\title{
Selling at the ultimate maximum in a regime-switching model
}

\author{
Yue Liu Nicolas Privault \\ School of Physical and Mathematical Sciences \\ Division of Mathematical Sciences \\ Nanyang Technological University \\ Singapore 637371
}

September 24, 2021

\begin{abstract}
This paper deals with optimal prediction in a regime-switching model driven by a continuous-time Markov chain. We extend existing results for geometric Brownian motion by deriving optimal stopping strategies that depend on the current regime state, and prove a number of continuity properties relating to optimal value and boundary functions. Our approach replaces the use of closed form expressions, which are not available in our setting, with PDE arguments that also simplify the approach of [2] in the classical Brownian case.
\end{abstract}

Key words: Optimal stopping; ultimate maximum; regime-switching models; free boundary problems; diffusion processes.

Mathematics Subject Classification (2010): 60G40; 35R35; 93E20; 60J28; $91 \mathrm{G} 80$.

\section{Introduction}

Regime-switching models have been introduced by Hamilton [6] in discrete time and are among the most popular and effective risky asset models. The regime-switching property is reflected in the changes of states of a Markov chain $\beta_{t}$, which stands for the influence of external market factors.

European options have been priced in continuous-time regime-switching models by Yao, Zhang and Zhou [15] via a recursive algorithm, and in Liu, Zhang and Yin [8] 
using the fast Fourier transform. Optimal stopping for option pricing in regimeswitching models has been considered in Guo [4], Guo and Zhang [5], Le and Wang [7], and in Ly Vath and Pham [14] with optimal switching. Optimal selling under threshold rules has been dealt with in Eloe et al. [3] in an exponential Gaussian diffusion model with regime switching. We refer to Shiryaev [12] and Peskir and Shiryaev [11] for background on the characterization of optimal stopping times and rewards.

The problem of selling a stock at the ultimate maximum has been considered by Du Toit and Peskir [2] as an extension of the results of Shiryaev, Xu and Zhou [13]. In this paper we extend the result of [2] to the framework of Markovian regime switching. Some of our results are natural extensions of those of [2] by averaging over the regimeswitching component, however the regime-switching case presents notable differences and additional difficulties compared with the classical Brownian case. For example, the optimal boundary functions depend on the regime state of the system, and they may not be monotone if the drift coefficients have switching signs. In addition we can no longer rely on closed form expressions as in [2] and instead we use PDE arguments, cf. e.g. Lemma 4.3, that also simplify the original approach.

In Lemma 2.1 we write the optimal value of the problem as a function of time, the regime state, and the relative maximum of the underlying asset. In the general case of real-valued drifts $\mu(i) \in \mathbb{R}, i \in \mathcal{M}$, we identify the optimal stopping time $\tau_{D}$ in Proposition 3.1, and in Proposition 3.2 we determine the structure of the optimal stopping set via its boundary functions $b_{D}(t, j)$ for $i$ in the state space $\mathcal{M}$ of the regime-switching chain.

When the drift parameters $(\mu(i))_{i \in \mathcal{M}}$ of the regime-switching chain are nonnegative we prove the continuity and monotonicity of boundary functions $b_{D}(t, j)$ in Proposition 5.1, by extending arguments of [2] to the regime-switching setting. Those results are illustrated in Figures 1 and 2 by the plotting of value functions that yield the optimal stopping boundaries. 
In Proposition 5.3 we show that immediate exercise is optimal when all drift parameters $\mu(i)$ are negative, $i \in \mathcal{M}$, while exercise at maturity becomes optimal when $\mu(i) \geq \sigma^{2}(i)$ for all $i \in \mathcal{M}$, where $\sigma(i)$ are the volatility parameters.

In Proposition 5.4 we derive a Volterra type integral equation (5.5) which is satisfied by the boundary function $b_{D}(t, j)$ of the stopping set. Such an equation is difficult to solve because, unlike in the classical setting [2], it also relies on the knowledge of the optimal value function, cf. Remark 5.5. In addition the associated free boundary problem (5.10a)-(5.10b) consists in a system of interacting PDEs that cannot be solved without additional assumptions, cf. e.g. Buffington and Elliott [1] for a solution under an ordering condition on boundary functions in the case of American options.

A treatment of drifts coefficients $(\mu(i))_{i \in \mathcal{M}}$ with switching signs has been proposed in of [9] via a recursive algorithm that does not rely on a Volterra equation. In this case it turns out that the boundary functions $b_{D}(t, i)$ may not be decreasing in $t \in[0, T]$.

We proceed as follows. In Section 2 we formulate the optimal prediction problem using optimal value functions. In Section 3 we derive the optimal stopping strategies in terms of the hitting time of the boundary function of a stopping set. Section 4 is devoted to continuity lemmas, which are used to prove the continuity of boundary functions. In Section 5 we also derive the Volterra integral equation which is satisfied by the boundary functions when the drift coefficients are nonnegative. Finally we study particular exercise strategies and we present a numerical simulation of boundary functions.

\section{Problem formulation}

Given a standard Brownian motion $\left(B_{t}\right)_{t \in \mathrm{R}_{+}}$independent of the Markov chain $\left(\beta_{t}\right)_{t \in \mathrm{R}_{+}}$, and the filtration $\left(\mathcal{F}_{t}\right)_{t \in \mathrm{R}_{+}}$generated by $\left(B_{t}\right)_{t \in \mathrm{R}_{+}}$and $\left(\beta_{t}\right)_{t \in \mathrm{R}_{+}}$, we consider an asset 
price $\left(Y_{t}\right)_{t \in \mathrm{R}_{+}}$modeled by a geometric Brownian motion

$$
d Y_{t}=\mu\left(\beta_{t}\right) Y_{t} d t+\sigma\left(\beta_{t}\right) Y_{t} d B_{t}, \quad 0 \leq t \leq T
$$

with regime switching driven by a finite-state continuous-time Markov chain $\left(\beta_{t}\right)_{t \in \mathrm{R}_{+}}$ on $\mathcal{M}=\{1,2, \ldots, m\}$, where $\mu: \mathcal{M} \longrightarrow \mathbb{R}$, and $\sigma: \mathcal{M} \longrightarrow(0, \infty)$ are deterministic functions. In this paper we deal with the optimal prediction problem

$$
V_{t}=\inf _{t \leq \tau \leq T} E\left[\sup _{0 \leq s \leq T} \frac{Y_{s}}{Y_{\tau}} \mid \mathcal{F}_{t}\right]
$$

introduced in [2] for geometric Brownian motion, in which the infimum of expected

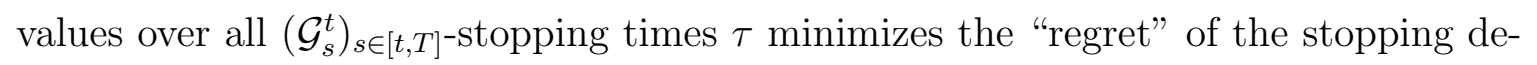
cision, where $\mathcal{G}_{s}^{t}:=\sigma\left(B_{r}-B_{t}, \beta_{r}: t \leq r \leq s\right), s \in[t, T]$.

The next Lemma 2.1 shows that the optimal value function $V_{t}$ in (2.2) can be written as a function of $\left(t, \beta_{t}, \hat{Y}_{0, t} / Y_{t}\right)$, where $\hat{Y}_{0, t}$ is defined by

$$
\hat{Y}_{t, s}:=\max _{t \leq r \leq s} Y_{r}, \quad 0 \leq t \leq s \leq T
$$

Lemma 2.1 The optimal value function $V_{t}$ in (2.2) takes the form

$$
V_{t}=V\left(t, \hat{Y}_{0, t} / Y_{t}, \beta_{t}\right)
$$

where the function $V:[0, T] \times[1,+\infty) \times \mathcal{M} \rightarrow \mathbb{R}_{+}$is given by

$$
V(t, x, j)=\inf _{t \leq \tau \leq T} E\left[\frac{1}{Y_{\tau}} \max \left(x Y_{t}, \hat{Y}_{t, T}\right) \mid \beta_{t}=j\right]
$$

$0 \leq t \leq T, x \geq 1, j \in \mathcal{M}$

Proof. Given $t \in[0, T]$, using the drifted Brownian motion

$$
\hat{B}_{u}^{t}:=B_{u+t}-B_{t}+\int_{t}^{t+u}\left(\frac{\mu\left(\beta_{s}\right)}{\sigma\left(\beta_{s}\right)}-\frac{\sigma\left(\beta_{s}\right)}{2}\right) d s, \quad u \in[0, T-t],
$$

we rewrite the solution of (2.1) as

$$
Y_{s}=Y_{t} \exp \left(\int_{0}^{s-t} \sigma\left(\beta_{u+t}\right) d \hat{B}_{u}^{t}\right), \quad s \in[t, T]
$$


and define

$$
\hat{S}_{s}^{t}:=\sup _{0 \leq r \leq s} \int_{0}^{r} \sigma\left(\beta_{u+t}\right) d \hat{B}_{u}^{t}, \quad s \in[0, T-t] .
$$

By the definition of $\hat{Y}_{t, s}$ in (2.3) and expression (2.5), and from the conditional independence of $\left(\left(\hat{B}_{r-t}^{t}\right)_{r \geq t},\left(\hat{S}_{r-t}^{t}\right)_{r \geq t}\right)$ with $\mathcal{F}_{t}$ given $\beta_{t}$ we have, for any $\left(\mathcal{G}_{s}^{t}\right)_{s \in[t, T]}$-stopping time $\tau$ with values in $[t, T]$, letting $a \vee b=\max (a, b)$,

$$
\begin{aligned}
& E\left[\sup _{0 \leq s \leq T} \frac{Y_{s}}{Y_{\tau}} \mid \mathcal{F}_{t}\right]=E\left[\frac{\hat{Y}_{0, t}}{Y_{\tau}} \vee \frac{\hat{Y}_{t, T}}{Y_{\tau}} \mid \mathcal{F}_{t}\right] \\
& =E\left[\left(\frac{\hat{Y}_{0, t}}{Y_{t}} \mathrm{e}^{-\int_{0}^{\tau-t} \sigma\left(\beta_{u+t}\right) d \hat{B}_{u}^{t}}\right) \vee \mathrm{e}^{\hat{S}_{T-t}^{t}-\int_{0}^{\tau-t} \sigma\left(\beta_{u+t}\right) d \hat{B}_{u}^{t}} \mid \mathcal{F}_{t}\right] \\
& =E\left[\left(\frac{\hat{Y}_{0, t}}{Y_{t}} \mathrm{e}^{-\int_{0}^{\tau-t} \sigma\left(\beta_{u+t}\right) d \hat{B}_{u}^{t}}\right) \vee \mathrm{e}^{\hat{S}_{T-t}^{t}-\int_{0}^{\tau-t} \sigma\left(\beta_{u+t}\right) d \hat{B}_{u}^{t}} \mid \beta_{t}, \frac{\hat{Y}_{0, t}}{Y_{t}}\right] \\
& =E\left[\frac{\hat{Y}_{0, t} \vee \hat{Y}_{t, T}}{Y_{\tau}} \mid \beta_{t}, \frac{\hat{Y}_{0, t}}{Y_{t}}\right] \\
& =E\left[\frac{1}{Y_{\tau}} \max \left(x Y_{t}, \hat{Y}_{t, T}\right) \mid \beta_{t}\right]_{x=\hat{Y}_{0, t} / Y_{t}}
\end{aligned}
$$

where the last line follows from the conditional independence between $\hat{Y}_{0, t} / Y_{t}$ and

$$
\left(\frac{Y_{\tau}}{Y_{t}}, \frac{\hat{Y}_{t, T}}{Y_{\tau}}\right)=\left(\exp \left(\int_{0}^{\tau-t} \sigma\left(\beta_{u+t}\right) d \hat{B}_{u}^{t}\right), \exp \left(\hat{S}_{T-t}^{t}-\int_{0}^{\tau-t} \sigma\left(\beta_{u+t}\right) d \hat{B}_{u}^{t}\right)\right)
$$

given $\beta_{t}$. Therefore by definition (2.2) and expression (2.4), we obtain

$$
\begin{aligned}
V_{t} & =\inf _{t \leq \tau \leq T} E\left[\sup _{0 \leq s \leq T} \frac{Y_{s}}{Y_{\tau}} \mid \mathcal{F}_{t}\right] \\
& =\inf _{t \leq \tau \leq T} E\left[\frac{1}{Y_{\tau}} \max \left(x Y_{t}, \hat{Y}_{t, T}\right) \mid \beta_{t}\right]_{x=\hat{Y}_{0, t} / Y_{t}} \\
& =V\left(t \frac{\hat{Y}_{0, t}}{Y_{t}}, \beta_{t},\right)
\end{aligned}
$$

In the next lemma we rewrite the optimal stopping problem (2.2) in the standard form (2.7) below, using the function

$$
G(t, x, i):=E\left[\max \left(x, \hat{Y}_{t, T} / Y_{t}\right) \mid \beta_{t}=i\right], \quad t \in[0, T], i \in \mathcal{M}, x \geq 1,
$$

with $G(T, x, i)=x, x \geq 1$. 
Lemma 2.2 The function $V:[0, T] \times[1,+\infty) \times \mathcal{M} \rightarrow \mathbb{R}_{+}$defined by (2.1) admits the expression

$$
V(t, x, j)=\inf _{t \leq \tau \leq T} E\left[G\left(\tau, X_{\tau}^{t, x}, \beta_{\tau}\right) \mid \beta_{t}=j\right],
$$

for $t \in[0, T], j \in \mathcal{M}, x \geq 1$, where

$$
X_{r}^{t, x}:=\frac{1}{Y_{r}} \max \left(x Y_{t}, \hat{Y}_{t, r}\right), \quad r \in[t, T], \quad x \geq 1 .
$$

Proof. By a conditional independence argument as in the proof of Lemma 2.1, for any $s \in[t, T]$ we have

$$
\begin{aligned}
E\left[\frac{\hat{Y}_{0, T}}{Y_{s}} \mid \mathcal{F}_{s}\right] & =E\left[\frac{\hat{Y}_{0, s} \vee \hat{Y}_{s, T}}{Y_{s}} \mid \mathcal{F}_{s}\right] \\
& =E\left[\frac{\hat{Y}_{0, s} \vee \hat{Y}_{s, T}}{Y_{s}} \mid \frac{\hat{Y}_{0, s}}{Y_{s}}, \beta_{s}\right] \\
& =E\left[y \vee \frac{\hat{Y}_{s, T}}{Y_{s}} \mid \beta_{s}\right]_{y=\hat{Y}_{0, s} / Y_{s}} \\
& =G\left(s, \frac{\hat{Y}_{0, s}}{Y_{s}}, \beta_{s}\right) .
\end{aligned}
$$

Next, we extend the above relation $(2.9)$ to $\left(\mathcal{G}_{s}^{t}\right)_{s \in[t, T]}$-stopping times $\tau$ written as the limit of a decreasing sequence of discrete stopping times by checking that for any discrete $\left(\mathcal{G}_{s}^{t}\right)_{s \in[t, T]}$-stopping time $\tau=\sum_{i=1}^{n} s_{i} \mathbf{1}_{\left\{\tau=s_{i}\right\}}, s_{1}, \ldots, s_{n} \in[t, T], n \geq 1$, by (2.9) we have

$$
\begin{aligned}
E\left[\frac{\hat{Y}_{0, T}}{Y_{\tau}} \mid \mathcal{F}_{\tau}\right] & =\sum_{i=1}^{n} E\left[\frac{\hat{Y}_{0, T}}{Y_{\tau}} \mathbf{1}_{\left\{\tau=s_{i}\right\}} \mid \mathcal{F}_{\tau}\right] \\
& =\sum_{i=1}^{n} E\left[\frac{\hat{Y}_{0, T}}{Y_{s_{i}}} \mathbf{1}_{\left\{\tau=s_{i}\right\}} \mid \mathcal{F}_{s_{i}}\right] \\
& =\sum_{i=1}^{n} E\left[\frac{\hat{Y}_{0, T}}{Y_{s_{i}}} \mid \mathcal{F}_{s_{i}}\right] \mathbf{1}_{\left\{\tau=s_{i}\right\}} \\
& =\sum_{i=1}^{n} G\left(s_{i}, \frac{\hat{Y}_{0, s_{i}}}{Y_{s_{i}}}, \beta_{s_{i}}\right) \mathbf{1}_{\left\{\tau=s_{i}\right\}} \\
& =G\left(\tau, \frac{\hat{Y}_{0, \tau}}{Y_{\tau}}, \beta_{\tau}\right) .
\end{aligned}
$$


Taking the conditional expectation $E\left[\cdot \mid \beta_{t}=j, \hat{Y}_{0, t} / Y_{t}=x\right]$ on both sides of the above equality, we obtain

$$
E\left[\frac{\hat{Y}_{0, T}}{Y_{\tau}} \mid \beta_{t}=j, \frac{\hat{Y}_{0, t}}{Y_{t}}=x\right]=E\left[G\left(\tau, \frac{\hat{Y}_{0, \tau}}{Y_{\tau}}, \beta_{\tau}\right) \mid \beta_{t}=j, \frac{\hat{Y}_{0, t}}{Y_{t}}=x\right] .
$$

By (2.8) and the conditional independence between $\hat{Y}_{0, t} / Y_{t}$ and $\left(Y_{t} / Y_{\tau}, \hat{Y}_{t, \tau} / Y_{\tau}\right)$ given $\beta_{t}=j$ we find

$$
\begin{gathered}
E\left[\frac{1}{Y_{\tau}} \max \left(x Y_{t}, \hat{Y}_{t, T}\right) \mid \beta_{t}=j\right]=E\left[\frac{\hat{Y}_{0, T}}{Y_{\tau}} \mid \beta_{t}=j, \frac{\hat{Y}_{0, t}}{Y_{t}}=x\right] \\
=E\left[G\left(\tau, \frac{\hat{Y}_{0, \tau}}{Y_{\tau}}, \beta_{\tau}\right) \mid \beta_{t}=j, \frac{\hat{Y}_{0, t}}{Y_{t}}=x\right] \\
=E\left[G\left(\tau, \frac{\left(x Y_{t}\right) \vee \hat{Y}_{t, \tau}}{Y_{\tau}}, \beta_{\tau}\right) \mid \beta_{t}=j, \frac{\hat{Y}_{0, t}}{Y_{t}}=x\right] \\
=E\left[G\left(\tau, X_{\tau}^{t, x}, \beta_{\tau}\right) \mid \beta_{t}=j\right],
\end{gathered}
$$

which completes the proof by (2.4).

\section{$3 \quad$ Stopping set and boundary functions}

In this section we apply Corollary 2.9 in [11] in the framework of the regime-switching model (2.1) with $\mu(i) \in \mathbb{R}, i \in \mathcal{M}$, in order to specify the stopping set and optimal stopping time associated to the optimal stopping problem (2.2), cf. Proposition 3.1 below. In order to deal with the existence of an optimal stopping time for (2.2) rewritten as (2.7), we define the set

$$
D:=\{(t, x, j) \in[0, T] \times[1, \infty) \times \mathcal{M}: V(t, x, j)=G(t, x, j)\} .
$$

From the relation $V(T, x, j)=G(T, x, j)=x, j \in \mathcal{M}, x \geq 1$, we check that $\{T\} \times$ $[1, \infty) \times \mathcal{M} \subset D$, which is consistent with the fact that the infimum in (2.2) is over $\left(\mathcal{G}_{s}^{t}\right)_{s \in[t, T]}$-stopping times $\tau \in[t, T]$. 
Proposition 3.1 Let $t \in[0, T]$. Given $\beta_{t}=j \in \mathcal{M}$ and $\hat{Y}_{0, t} / Y_{t}=x \in[1, \infty)$, the $\left(\mathcal{G}_{s}^{t}\right)_{s \in[t, T]^{-s t o p p i n g ~ t i m e ~}}$

$$
\tau_{D}(t, x, j):=\inf \left\{r \geq t:\left(r, \frac{\hat{Y}_{0, r}}{Y_{r}}, \beta_{r}\right) \in D\right\}
$$

is an optimal stopping time for (2.2), or equivalently for (2.7), provided it is a.s. finite.

Proof. By Corollary 2.9 in [11] the optimal stopping time for problem (2.7) exists and is equal to $\tau_{D}(t, x, j)$ in (3.2) provided we check that for all $t \in[0, T]$ we have:

a) $G(t, x, j)$ is lower semicontinuous with respect to $x$, as follows directly from the definition (2.6) of $G(t, x, j)$.

b) $V(t, x, j)$ is upper semicontinuous with respect to $x$, as follows from the continuity Lemma 4.5 below.

c) We have $E\left[\sup _{t \leqslant s \leqslant T}\left|G\left(s, X_{s}^{t, x}, \beta_{s}\right)\right|\right]<\infty$. Indeed, from (2.8) and (2.5) we have

$$
\begin{aligned}
X_{s}^{t, x} & =\frac{1}{Y_{s}} \max \left(x Y_{t}, \hat{Y}_{t, s}\right) \\
& =Y_{t}^{-1} \mathrm{e}^{-\int_{0}^{s-t} \sigma\left(\beta_{u+t}\right) d \hat{B}_{u}^{t}} \max \left(x Y_{t}, Y_{t} \mathrm{e}^{\hat{S}_{s-t}^{t}}\right) \\
& =\mathrm{e}^{\max \left(\log x, \hat{S}_{s-t}^{t}\right)-\int_{0}^{s-t} \sigma\left(\beta_{u+t}\right) d \hat{B}_{u}^{t}}, \quad s \in[t, T] .
\end{aligned}
$$

Hence by (2.6) and the conditional independence between $X_{s}^{t, x}=\max \left(x Y_{t} / Y_{s}, \hat{Y}_{t, s} / Y_{s}\right)$ and $\hat{Y}_{s, T} / Y_{s}$ given $\beta_{s}$, we find that

$$
\begin{aligned}
& G\left(s, X_{s}^{t, x}, \beta_{s}\right)=E\left[y \vee \frac{\hat{Y}_{s, T}}{Y_{s}} \mid \beta_{s}\right]_{y=X_{s}^{t, x}} \\
& =E\left[X_{s}^{t, x} \vee \frac{\hat{Y}_{s, T}}{Y_{s}} \mid \beta_{s}, X_{s}^{t, x}\right] \\
& \quad=E\left[\mathrm{e}^{-\int_{0}^{s-t} \sigma\left(\beta_{u+t}\right) d \hat{B}_{u}^{t}}\left(\mathrm{e}^{\max \left(\log x, \sup _{t \leq r \leq s} \int_{0}^{r-t} \sigma\left(\beta_{u+t}\right) d \hat{B}_{u}^{t}\right)} \vee \mathrm{e}^{\sup _{s \leq r \leq T} \int_{0}^{r-t} \sigma\left(\beta_{u+t}\right) d \hat{B}_{u}^{t}}\right) \mid \beta_{s}, X_{s}^{t, x}\right] \\
& =E\left[\mathrm{e}^{\max \left(\log x, \hat{S}_{T-t}^{t}\right)-\int_{0}^{s-t} \sigma\left(\beta_{u+t}\right) d \hat{B}_{u}^{t}} \mid \beta_{s}, X_{s}^{t, x}\right] .
\end{aligned}
$$

Letting

$$
\check{S}_{T-t}^{t}:=\inf _{t \leqslant s \leqslant T} \int_{0}^{s-t} \sigma\left(\beta_{u+t}\right) d \hat{B}_{u}^{t}
$$




$$
=\inf _{t \leqslant s \leqslant T}\left[\int_{0}^{s-t} \sigma\left(\beta_{u+t}\right) d B_{t+u}+\int_{0}^{s-t}\left(\mu\left(\beta_{u+t}\right)-\sigma^{2}\left(\beta_{u+t}\right) / 2\right) d u\right]
$$

we conclude that

$$
\begin{aligned}
E & {\left[\sup _{t \leqslant s \leqslant T}\left|G\left(s, X_{s}^{t, x}, \beta_{s}\right)\right|\right]=E\left[\sup _{t \leqslant s \leqslant T} E\left[\mathrm{e}^{\max \left(\log x, \hat{S}_{T-t}^{t}\right)-\int_{0}^{s-t} \sigma\left(\beta_{u+t}\right) d \hat{B}_{u}^{t}} \mid \beta_{s}, X_{s}^{t, x}\right]\right] } \\
& \leq x E\left[\sup _{t \leqslant s \leqslant T} E\left[\mathrm{e}^{\hat{S}_{T-t}^{t}-\int_{0}^{s-t} \sigma\left(\beta_{u+t}\right) d \hat{B}_{u}^{t}} \mid \beta_{s}, X_{s}^{t, x}\right]\right] \\
& \leq x E\left[\sup _{t \leqslant s \leqslant T} E\left[\mathrm{e}^{\hat{S}_{T-t}^{t}-\inf _{t \leqslant r \leqslant T} \int_{0}^{r-t} \sigma\left(\beta_{u+t}\right) d \hat{B}_{u}^{t}} \mid \beta_{s}, X_{s}^{t, x}\right]\right] \\
& =x E\left[\mathrm{e}^{\hat{S}_{T-t}^{t}-\inf _{t \leqslant r \leqslant T} \int_{0}^{r-t} \sigma\left(\beta_{u+t}\right) d \hat{B}_{u}^{t}}\right] \\
& =x E\left[\mathrm{e}^{\hat{S}_{T-t}^{t}-\check{S}_{T-t}^{t}}\right] \\
& \leq x \sqrt{E\left[\mathrm{e}^{2 \hat{S}_{T-t}^{t}}\right] E\left[\mathrm{e}^{-2 \check{S}_{T-t}^{t}}\right]} \\
& \leq x \mathrm{e}^{\max _{i \in \mathcal{M}}\left|\sigma^{2}(i)-2 \mu(i)\right|(T-t)} \sqrt{E\left[\mathrm { e } ^ { 2 \hat { S } _ { T - t } ^ { t } ] } E \left[\mathrm{e}^{\left.2 \hat{S}_{T-t}^{t}\right]}\right.\right.} \\
& \leq x E\left[\mathrm{e}^{\left.2 \hat{S}_{T-t}^{t}\right]} \mathrm{e}^{\max _{i \in \mathcal{M}}\left|\sigma^{2}(i)-2 \mu(i)\right|(T-t)}\right. \\
& <\infty .
\end{aligned}
$$

Define

$$
F(t, x, j):=V(t, x, j)-G(t, x, j) \leq 0,
$$

which is nonpositive by $(2.7), t \in[0, T], j \in \mathcal{M}, x \geq 1$, so that we have

$$
D=\{(t, x, j) \in[0, T] \times[1, \infty) \times \mathcal{M}: F(t, x, j)=0\}
$$

hence $D$ is closed from the continuity of $(t, x) \longmapsto V(t, x, j)$ and $(t, x) \longmapsto G(t, x, j)$ on $[0, T] \times[1, \infty)$, cf. Lemmas 4.5 and Lemmas 4.6 below, respectively. The continuation set $C=D^{c}$ is an open set that can be written as

$$
C=\{(t, x, j) \in[0, T] \times[1, \infty) \times \mathcal{M}: F(t, x, j)<0\}
$$

In the next Proposition 3.2 we characterize the shape of the stopping set $D$ defined in $(3.1)$ in terms of the boundary function $b_{D}(t, j)$ defined by

$$
b_{D}(t, j):=\inf \{x \in[1, \infty):(t, x, j) \in D\} .
$$


From the relation $\{T\} \times[1, \infty) \times \mathcal{M} \subset D$ we deduce the terminal condition $b_{D}(T, j)=$ $1, j \in \mathcal{M}$.

Proposition 3.2 For any $(t, x, j) \in[0, T] \times[1, \infty) \times \mathcal{M}$ such that $(t, x, j) \in D$ we have

$$
\{t\} \times[x, \infty) \times\{j\} \subset D .
$$

and

$$
D=\left\{(t, y, j) \in[0, T] \times[1, \infty) \times \mathcal{M}: y \geq b_{D}(t, j)\right\} .
$$

Proof. Let $y:=\sup \{z \in[x, \infty):\{t\} \times[x, z] \times\{j\} \subset D\}$. If $y<\infty$ then we have $(t, y, j) \in D$ by the closedness of $D$, and from the monotonicity property of $F(t, x, j)$ stated in Lemma 3.3, $(t, y, j) \in D$ admits a right neighborhood of the form

$$
\{t\} \times[x, x+\eta] \times\{j\} \subset D
$$

for some $\eta>0$, which leads to a contradiction. Hence $y=+\infty$ and (3.9) holds. Relation (3.10) follows from the equivalence

$$
(t, x, j) \in D \Longleftrightarrow\{t\} \times[x, \infty) \times\{j\} \subset D \Longleftrightarrow x \geq b_{D}(t, j)
$$

that follows from (3.8).

The following lemma has been used in the proof of Proposition 3.2.

Lemma 3.3 For any $(t, x, j) \in D$, we have

$$
\liminf _{\varepsilon \searrow 0} \frac{F(t, x+\varepsilon, j)-F(t, x, j)}{\varepsilon} \geq 0 .
$$

Proof. We split the proof into two parts.

(i) From (3.4) we have

$$
\begin{aligned}
G\left(s, X_{s}^{t, x}, \beta_{s}\right) & =E\left[X_{s}^{t, x} \vee \frac{\hat{Y}_{s, T}}{Y_{s}} \mid \beta_{s}, X_{s}^{t, x}\right]=E\left[X_{s}^{t, x} \vee \frac{\hat{Y}_{s, T}}{Y_{s}} \mid \mathcal{F}_{s}\right] \\
& =E\left[\mathrm{e}^{\max \left(\log x, \hat{S}_{T-t}^{t}\right)-\int_{0}^{s-t} \sigma\left(\beta_{u+t}\right) d \hat{B}_{u}^{t}} \mid \mathcal{F}_{s}\right], \quad s \in[t, T],
\end{aligned}
$$

which extends to any $\left(\mathcal{G}_{s}^{t}\right)_{s \in[t, T]}$-stopping time $\tau \in[t, T]$ as

$$
G\left(\tau, X_{\tau}^{t, x}, \beta_{\tau}\right)=E\left[\mathrm{e}^{\max \left(\log x, \hat{S}_{T-t}^{t}\right)-\int_{0}^{\tau-t} \sigma\left(\beta_{u+t}\right) d \hat{B}_{u}^{t}} \mid \mathcal{F}_{\tau}\right],
$$


as in (2.9)-(2.10) above. For all $x \geq 1$ and $\varepsilon>0$, consider the $\left(\mathcal{G}_{s}^{t}\right)_{s \in[t, T]}$-stopping time

$$
\tau_{\varepsilon}^{+}:=\tau_{D}(t, x+\varepsilon, j) \in[t, T]
$$

defined by (3.2), which solves the optimal stopping problem

$V(t, x+\varepsilon, j)=\inf _{t \leq \tau \leq T} E\left[G\left(\tau, X_{\tau}^{t, x+\varepsilon}, \beta_{\tau}\right) \mid \beta_{t}=j\right]=E\left[G\left(\tau_{\varepsilon}^{+}, X_{\tau_{\varepsilon}^{+}}^{t, x+\varepsilon}, \beta_{\tau_{\varepsilon}^{+}}\right) \mid \beta_{t}=j\right]$,

cf. (2.7). The following argument relies on the fact that for any $(t, x, j) \in D$ we have

$$
\lim _{\varepsilon \rightarrow 0} \tau_{D}(t, x+\varepsilon, j)=t,
$$

as will be shown in part (ii) below. Relations (2.6), (2.7), (3.12) and (3.13) imply

$$
\begin{aligned}
\liminf _{\varepsilon \searrow 0} \frac{V(t, x+\varepsilon, j)-V(t, x, j)}{\varepsilon} \\
\geq \liminf _{\varepsilon \searrow 0} \frac{1}{\varepsilon} E\left[G\left(\tau_{\varepsilon}^{+}, X_{\tau_{\varepsilon}^{+}}^{t, x+\varepsilon}, \beta_{\tau_{\varepsilon}^{+}}\right)-G\left(\tau_{\varepsilon}^{+}, X_{\tau_{\varepsilon}^{+}}^{t, x}, \beta_{\tau_{\varepsilon}^{+}}\right) \mid \beta_{t}=j\right] \\
=\liminf _{\varepsilon \searrow 0} \frac{1}{\varepsilon} E\left[E\left[\mathrm{e}^{\log (x+\varepsilon) \vee \hat{S}_{T-t}^{t}-\int_{0}^{\tau_{\varepsilon}^{+}-t} \sigma\left(\beta_{u+t}\right) d \hat{B}_{u}^{t}}-\mathrm{e}^{\log x \vee \hat{S}_{T-t}^{t}-\int_{0}^{\tau_{\varepsilon}^{+}-t} \sigma\left(\beta_{u+t}\right) d \hat{B}_{u}^{t}} \mid \mathcal{F}_{\tau_{\varepsilon}^{+}}\right] \mid \beta_{t}=j\right] \\
=\liminf _{\varepsilon \searrow 0} \frac{1}{\varepsilon} E\left[\mathrm{e}^{\log (x+\varepsilon) \vee \hat{S}_{T-t}^{t}-\int_{0}^{\tau_{\varepsilon}^{+}-t} \sigma\left(\beta_{u+t}\right) d \hat{B}_{u}^{t}}-\mathrm{e}^{\log x \vee \hat{S}_{T-t}^{t}-\int_{0}^{\tau_{\varepsilon}^{+}-t} \sigma\left(\beta_{u+t}\right) d \hat{B}_{u}^{t}} \mid \beta_{t}=j\right] \\
=\liminf _{\varepsilon \searrow 0} \frac{1}{\varepsilon} E\left[\mathrm{e}^{\log (x+\varepsilon) \vee \hat{S}_{T-t}^{t}}-\mathrm{e}^{\log x \vee \hat{S}_{T-t}^{t}} \mid \beta_{t}=j\right] \\
=\frac{\partial}{\partial x} E\left[\mathrm{e}^{\max \left(\log x, \hat{S}_{T-t}^{t}\right)} \mid \beta_{t}=j\right] \\
=\frac{\partial G}{\partial x}(t, x, j),
\end{aligned}
$$

hence we conclude to (3.11). Here we used the dominated convergence theorem with the bound

$$
\begin{array}{r}
\frac{1}{\varepsilon}\left|\mathrm{e}^{\log (x+\varepsilon) \vee \hat{S}_{T-t}^{t}-\int_{0}^{\tau_{\varepsilon}^{+}-t} \sigma\left(\beta_{u+t}\right) d \hat{B}_{u}^{t}}-\mathrm{e}^{\log x \vee \hat{S}_{T-t}^{t}-\int_{0}^{\tau_{\varepsilon}^{+}-t} \sigma\left(\beta_{u+t}\right) d \hat{B}_{u}^{t}}\right| \\
\leq\left|\frac{\mathrm{e}^{\log (x+\varepsilon)}-\mathrm{e}^{\log x}}{\varepsilon}\right| \mathrm{e}^{-\inf _{0 \leq s \leq T-t} \int_{0}^{s} \sigma\left(\beta_{t+u}\right) d \hat{B}_{u}^{t}}=\mathrm{e}^{-\breve{S}_{T-t}^{t},}
\end{array}
$$

where $\check{S}_{T-t}^{t}$ is defined in (3.5) and the righthand side is integrable as in the derivation of (3.6).

(ii) We turn to the proof of (3.13). From the expression (2.4) in Lemma 2.1, we have

$$
V(t, x, j)=\inf _{t \leq \tau \leq T} E\left[\frac{x Y_{t} \vee \hat{Y}_{t, T}}{Y_{\tau}} \mid \beta_{t}=j, \mathcal{F}_{t}\right]
$$




$$
=\inf _{t \leq \tau \leq T} E\left[\mathrm{e}^{-\int_{0}^{\tau-t} \sigma\left(\beta_{u+t}\right) d \hat{B}_{u}^{t}}\left(x \vee \mathrm{e}^{\hat{S}_{T-t}^{t}}\right) \mid \beta_{t}=j, \mathcal{F}_{t}\right] .
$$

From (3.15) and

$$
X_{r}^{t, x+\varepsilon}=\mathrm{e}^{-\int_{0}^{r-t} \sigma\left(\beta_{u+t}\right) d \hat{B}_{u}^{t}}\left(x+\varepsilon \vee \mathrm{e}^{\hat{S}_{r-t}^{t}}\right)
$$

cf. (3.3), we obtain

$$
\begin{aligned}
V\left(r, X_{r}^{t, x+\varepsilon}, \beta_{r}\right) & =\inf _{r \leq \tau \leq T} E\left[\mathrm{e}^{-\int_{0}^{\tau-r} \sigma\left(\beta_{u+r}\right) d \hat{B}_{u}^{r}}\left(y \vee \mathrm{e}^{\hat{S}_{T-r}^{r}}\right) \mid \mathcal{F}_{r}\right]_{y=X_{r}^{t, x+\varepsilon}} \\
& =\inf _{r \leq \tau \leq T} E\left[\mathrm{e}^{-\int_{0}^{\tau-r} \sigma\left(\beta_{u+r}\right) d \hat{B}_{u}^{r}}\left(X_{r}^{t, x+\varepsilon} \vee \mathrm{e}^{\hat{S}_{T-r}^{r}}\right) \mid \mathcal{F}_{r}\right]
\end{aligned}
$$

Next, from the definition (3.2) of $\tau_{D}(t, x+\varepsilon, j)$ and (3.16) we have, on the event

$$
\begin{aligned}
& \left\{\beta_{t}=j\right\}, \\
& \tau_{D}(t, x+\varepsilon, j)=\inf \left\{r \geq t:\left(r, X_{r}^{t, x+\varepsilon}, \beta_{r}\right) \in D\right\} \\
& \quad=\inf \left\{r \geq t: \inf _{r \leq \tau \leq T} E\left[\mathrm{e}^{-\int_{0}^{\tau-r} \sigma\left(\beta_{u+r}\right) d \hat{B}_{u}^{r}}\left(X_{r}^{t, x+\varepsilon} \vee \mathrm{e}^{\hat{S}_{T-r}^{r}}\right) \mid \mathcal{F}_{r}\right]=E\left[X_{r}^{t, x+\varepsilon} \vee \mathrm{e}^{\hat{S}_{T-r}^{r}} \mid \mathcal{F}_{r}\right]\right\} \\
& \quad \leq \inf \left\{r \geq t: \inf _{r \leq \tau \leq T} E\left[\mathrm{e}^{-\int_{0}^{\tau-r} \sigma\left(\beta_{u+r}\right) d \hat{B}_{u}^{r}}\left(X_{r}^{t, x} \vee \mathrm{e}^{\hat{S}_{T-r}^{r}}\right) \mid \mathcal{F}_{r}\right] \geq E\left[X_{r}^{t, x+\varepsilon} \vee \mathrm{e}^{\hat{S}_{T-r}^{r}} \mid \mathcal{F}_{r}\right]\right\} \\
& \leq \inf \left\{r \geq t: \inf _{r \leq \tau \leq T} E\left[\mathrm{e}^{-\int_{0}^{\tau-r} \sigma\left(\beta_{u+r}\right) d \hat{B}_{u}^{r}}\left(X_{r}^{t, x} \vee \mathrm{e}^{\hat{S}_{T-r}^{r}}\right) \mid \mathcal{F}_{r}\right] \geq \mathrm{e}^{\varepsilon} E\left[X_{r}^{t, x} \vee \mathrm{e}^{\hat{S}_{T-r}^{r}} \mid \mathcal{F}_{r}\right]\right\},
\end{aligned}
$$

where we applied the inequality

$$
\begin{aligned}
& X_{r}^{t, x+\varepsilon}=\mathrm{e}^{-\int_{0}^{r-t} \sigma\left(\beta_{u+t}\right) d \hat{B}_{u}^{t}}\left(\mathrm{e}^{\log (x+\varepsilon)} \vee \mathrm{e}^{\hat{S}_{r-t}^{t}}\right) \leq \mathrm{e}^{-\int_{0}^{r-t} \sigma\left(\beta_{u+t}\right) d \hat{B}_{u}^{t}+\varepsilon}\left(\mathrm{e}^{\log (x)} \vee \mathrm{e}^{\hat{S}_{r-t}^{t}}\right)=\mathrm{e}^{\varepsilon} X_{r}^{t, x}, \\
& x \geq 1, \varepsilon \geq 0, r \in[t, T] . \text { This implies } \\
& \lim _{\varepsilon \rightarrow 0} \tau_{D}(t, x+\varepsilon, j) \\
& \leq \liminf _{\varepsilon \rightarrow 0}\left\{r \geq t: \inf _{r \leq \tau \leq T} E\left[\mathrm{e}^{-\int_{0}^{\tau-r} \sigma\left(\beta_{u+r}\right) d \hat{B}_{u}^{r}}\left(X_{r}^{t, x} \vee \mathrm{e}_{T-r}^{\hat{S}_{T-r}^{r}}\right) \mid \mathcal{F}_{r}\right] \geq \mathrm{e}^{\varepsilon} E\left[X_{r}^{t, x} \vee \mathrm{e}^{\hat{S}_{T-r}^{r}} \mid \mathcal{F}_{r}\right]\right\} \\
& =\inf \left\{r \geq t: \inf _{r \leq \tau \leq T} E\left[\mathrm{e}^{-\int_{0}^{\tau-r} \sigma\left(\beta_{u+r}\right) d \hat{B}_{u}^{r}}\left(X_{r}^{t, x} \vee \mathrm{e}^{\hat{S}_{T-r}^{r}}\right) \mid \mathcal{F}_{r}\right] \geq E\left[X_{r}^{t, x} \vee \mathrm{e}^{\hat{S}_{T-r}^{r}} \mid \mathcal{F}_{r}\right]\right\} \\
& =\inf \left\{r \geq t: \inf _{r \leq \tau \leq T} E\left[\mathrm{e}^{-\int_{0}^{\tau-r} \sigma\left(\beta_{u+r}\right) d \hat{B}_{u}^{r}}\left(X_{r}^{t, x} \vee \mathrm{e}^{\hat{S}_{T-r}^{r}}\right) \mid \mathcal{F}_{r}\right]=E\left[X_{r}^{t, x} \vee \mathrm{e}^{\hat{S}_{T-r}^{r}} \mid \mathcal{F}_{r}\right]\right\} \\
& =\inf \left\{r \geq t:\left(r, X_{r}^{t, x}, \beta_{r}\right) \in D\right\} \\
& =t,
\end{aligned}
$$

since $(t, x, j) \in D, \beta_{t}=j$ and $X_{t}^{t, x}=x$. Since $\tau_{D}(t, x+\varepsilon, j) \geq t$ we conclude to (3.13). 


\section{Continuity lemmas}

The following property of smooth fit, namely the continuity of the function $y \longmapsto$ $\frac{\partial V}{\partial y}(t, y, j)$ over the optimal stopping boundary $\partial C$, will be needed in the proof of Proposition 5.4 below.

Lemma 4.1 For any $(t, y, j) \in \partial C, y>1$, we have

$$
\frac{\partial V}{\partial y}(t, y+, j)=\frac{\partial V}{\partial y}(t, y-, j) .
$$

Proof. For any $\varepsilon \in(0, y-1)$, let $\tau_{\varepsilon}^{-}=\tau_{D}(t, y-\varepsilon, j) \in[t, T]$, cf. (3.2). Since $(t, y, j) \in \partial C$ and $D$ is closed we have $(t, y, j) \in D$. Similarly to (3.17) to (3.18), $\tau_{\varepsilon}^{-}$ converges to $t$ a.s. when $\varepsilon$ tends to 0 . By the same approach as in (3.14), replacing $y+\varepsilon$ with $y-\varepsilon$ shows that

$$
\frac{\partial G}{\partial y}(t, y, j) \leq \liminf _{\varepsilon \searrow 0} \frac{V(t, y-\varepsilon, j)-V(t, y, j)}{\varepsilon} .
$$

On the other hand, since $(t, y, j) \in \partial C \subset D$, we have

$$
\limsup _{\varepsilon \searrow 0} \frac{V(t, y-\varepsilon, j)-V(t, y, j)}{\varepsilon} \leq \lim _{\varepsilon \searrow 0} \frac{G(t, y-\varepsilon, j)-G(t, y, j)}{\varepsilon}=\frac{\partial G}{\partial y}(t, y, j)
$$

hence

$$
\frac{\partial V}{\partial y}(t, y-, j)=\frac{\partial G}{\partial y}(t, y, j)
$$

Finally the fact that $V=G$ on the closed set $D$ implies

$$
\frac{\partial V}{\partial y}(t, y-, j)=\frac{\partial V}{\partial y}(t, y+, j)=\frac{\partial G}{\partial y}(t, y, j) .
$$

In the next proposition, which will be used in the proof of Proposition 5.4, we show the normal reflection of the free boundary problem by proving that the right derivative of the value function $V(t, y, j)$ vanishes at $y=1$, cf. also page 264 of [11] without regime switching.

Lemma 4.2 For any $t \in[0, T]$ and $j \in \mathcal{M}$ we have

$$
\frac{\partial V}{\partial y}(t, 1+, j)=0
$$


Proof. For convenience of notation we set $\tau_{0}=\tau_{D}(t, 1, j)$, and note that

$$
\begin{aligned}
& \limsup _{\varepsilon \searrow 0} \frac{V(t, 1+\varepsilon, j)-V(t, 1, j)}{\varepsilon} \\
& \leq \limsup _{\varepsilon \searrow 0} \frac{1}{\varepsilon} E\left[G\left(\tau_{0}, X_{\tau_{0}}^{t, 1+\varepsilon}, \beta_{\tau_{0}}\right)-G\left(\tau_{0}, X_{\tau_{0}}^{t, 1}, \beta_{\tau_{0}}\right) \mid \beta_{t}=j\right] \\
& =\underset{\varepsilon \searrow 0}{\limsup } \frac{1}{\varepsilon} E\left[\mathrm{e}^{\log (1+\varepsilon) \vee \hat{S}_{T-t}^{t}-\int_{0}^{\tau_{0}-t} \sigma\left(\beta_{t+r}\right) d \hat{B}_{r}^{t}}-\mathrm{e}^{\hat{S}_{T-t}^{t}-\int_{0}^{\tau_{0}-t} \sigma\left(\beta_{t+r}\right) d \hat{B}_{r}^{t}} \mid \beta_{t}=j\right] \\
& =\underset{\varepsilon \searrow 0}{\limsup } E\left[\frac{1}{\varepsilon}\left(\mathrm{e}^{\log (1+\varepsilon) \vee \hat{S}_{T-t}^{t}-\int_{0}^{\tau_{0}-t} \sigma\left(\beta_{t+r}\right) d \hat{B}_{r}^{t}}-\mathrm{e}^{\hat{S}_{T-t}^{t}-\int_{0}^{\tau_{0}-t} \sigma\left(\beta_{t+r}\right) d \hat{B}_{r}^{t}}\right) \mathbf{1}_{\left\{\hat{S}_{T-t}^{t}<\log (1+\varepsilon)\right\} \mid} \mid \beta_{t}=j\right] \\
& =E\left[\limsup _{\varepsilon \searrow 0} \frac{\mathrm{e}^{\log (1+\varepsilon) \vee \hat{S}_{T-t}^{t}-\int_{0}^{\tau_{0}-t} \sigma\left(\beta_{t+r}\right) d \hat{B}_{r}^{t}}-\mathrm{e}^{\hat{S}_{T-t}^{t}-\int_{0}^{\tau_{0}-t} \sigma\left(\beta_{t+r}\right) d \hat{B}_{r}^{t}}}{\varepsilon} \mathbf{1}_{\left\{\hat{S}_{T-t}^{t}<\log (1+\varepsilon)\right\} \mid} \mid \beta_{t}=j\right] \\
& =0, \quad
\end{aligned}
$$

since $\lim _{\varepsilon \searrow 0} \mathbf{1}_{\left\{\hat{S}_{T-t}^{t}<\log (1+\varepsilon)\right\}}=0$, where we applied the dominated convergence theorem as in the proof of Lemma 3.3 with the same dominating function as in (3.15). Since $V(t, y, j)$ is nondecreasing in $y \in[1, \infty)$, we have

$$
\liminf _{\varepsilon \searrow 0} \frac{V(t, 1+\varepsilon, j)-V(t, 1, j)}{\varepsilon} \geq 0,
$$

which shows that

$$
\frac{\partial V}{\partial y}(t, 1+, j)=\lim _{\varepsilon \searrow 0} \frac{V(t, 1+\varepsilon, j)-V(t, 1, j)}{\varepsilon}=0 .
$$

Next we consider the infinitesimal generator

$$
\mathbb{L} f(s, x, j)=\left(\frac{\partial}{\partial s}+x\left(\sigma^{2}(j)-\mu(j)\right) \frac{\partial}{\partial x}+\frac{1}{2} \sigma^{2}(j) x^{2} \frac{\partial^{2}}{\partial x^{2}}\right) f(s, x, j)+\sum_{i=1}^{m} q_{j, i} f(s, x, i),
$$

of the Markov process $\left(s, X_{s}^{t, x}, \beta_{s}\right)_{s \in[t, T]}$, where $Q=\left(q_{i j}\right)_{i, j=1, \ldots, m}$ is the infinitesimal matrix generator of the Markov process $\left(\beta_{t}\right)_{t \in[0, T]}$, for any sufficiently differentiable function $f$ of $(s, y, j) \in[0, T] \times[1, \infty) \times \mathcal{M}$, cf. Lemma 4.7 below.

The following lemmas will be used in the proof of Proposition 5.3 below. In Lemma 4.3 we replace the use of closed form expressions for $\mathbb{L} G(t, x, j)$, which are no longer available in our setting, with the differential expression (4.3). 
Lemma 4.3 We have

$$
\frac{\partial G}{\partial x}(t, 1+, j)=0, \quad t \in[0, T]
$$

and

$$
\mathbb{L} G(t, x, j)=x \sigma^{2}(j) \frac{\partial G}{\partial x}(t, x, j)-\mu(j) G(t, x, j), \quad t \in[0, T],
$$

with $\mathbb{L} G(T, x, j)=-\mu(j) x, j \in \mathcal{M}, x \in[1, \infty)$. In particular, for any $(t, x, j) \in$ $[0, T) \times[1, \infty) \times \mathcal{M}$ we have

$$
\left\{\begin{array}{l}
\mathbb{L} G(t, x, j)>0, \text { when } \mu(j) \leq 0 \\
\mathbb{L} G(t, x, j)<0, \text { when } \mu(j) \geq \sigma^{2}(j)
\end{array}\right.
$$

In addition, $\mathbb{L} G(t, x, j)$ is nondecreasing and continuous in $t$ for all $x \geq 1$ when $\mu(j) \geq 0$.

Proof. For all $j \in \mathcal{M}$ we let

$$
f(t, y, z, j):=y G\left(t, \frac{z}{y}, j\right)=E\left[\max \left(z, y \frac{\hat{Y}_{t, T}}{Y_{t}}\right) \mid \beta_{t}=j\right], \quad t \in[0, T], \quad y, z>0 .
$$

By (2.1) and the Itô formula we have

$$
\begin{aligned}
d f\left(t, Y_{t}, \hat{Y}_{0, t}, \beta_{t}\right)= & \frac{\partial f}{\partial t}\left(t, Y_{t}, \hat{Y}_{0, t}, \beta_{t}\right) d t+\mu\left(\beta_{t}\right) Y_{t} \frac{\partial f}{\partial x}\left(t, Y_{t}, \hat{Y}_{0, t}, \beta_{t}\right) d t \\
& +\sigma\left(\beta_{t}\right) Y_{t} \frac{\partial f}{\partial x}\left(t, Y_{t}, \hat{Y}_{0, t}, \beta_{t}\right) d B_{t}+\frac{1}{2} \sigma^{2}\left(\beta_{t}\right) Y_{t}^{2} \frac{\partial^{2} f}{\partial x^{2}}\left(t, Y_{t}, \hat{Y}_{0, t}, \beta_{t}\right) d t \\
& +\frac{\partial f}{\partial y}\left(t, Y_{t}, \hat{Y}_{0, t}, \beta_{t}\right) d \hat{Y}_{0, t}+f\left(t, Y_{t}, \hat{Y}_{0, t}, \beta_{t}\right)-f\left(t, Y_{t}, \hat{Y}_{0, t}, \beta_{t^{-}}\right)
\end{aligned}
$$

and given that

$$
f\left(t, Y_{t}, \hat{Y}_{0, t}, \beta_{t}\right)=E\left[\hat{Y}_{0, T} \mid \beta_{t}, Y_{t}, \hat{Y}_{0, t}\right]=E\left[\hat{Y}_{0, T} \mid \mathcal{F}_{t}\right], \quad t \in[0, T]
$$

is a martingale and $\left(\hat{Y}_{0, t}\right)_{t \in[0, T]}$ has finite variation, we find

$$
\frac{\partial f}{\partial t}(t, y, z, j)+\mu(j) y \frac{\partial f}{\partial x}(t, y, z, j)+\frac{1}{2} \sigma^{2}(j) y^{2} \frac{\partial^{2} f}{\partial x^{2}}(t, y, z, j)+\sum_{i=1}^{m} q_{j, i} f(t, y, z, i)=0
$$


and $\frac{\partial f}{\partial y}(t, x, y, j)_{x=y}=0$. Substituting (4.5) into (4.6) shows that

$$
\begin{aligned}
& y \frac{\partial G}{\partial t}\left(t, \frac{z}{y}, j\right)+\mu(j) y\left(G\left(t, \frac{z}{y}, j\right)+y \frac{\partial G}{\partial x}\left(t, \frac{z}{y}, j\right)\left(-\frac{z}{y^{2}}\right)\right) \\
& +\frac{1}{2} \sigma^{2}(j) y^{2}\left(\frac{\partial G}{\partial x}\left(t, \frac{z}{y}, j\right)\left(-\frac{z}{y^{2}}\right)+\frac{z}{y^{2}} \frac{\partial G}{\partial x}\left(t, \frac{z}{y}, j\right)+\frac{z^{2}}{y^{3}} \frac{\partial^{2} G}{\partial x^{2}}\left(t, \frac{z}{y}, j\right)\right) \\
& +\sum_{i=1}^{m} q_{j, i} y G\left(t, \frac{z}{y}, i\right)=0,
\end{aligned}
$$

which shows that the function $G(t, x, j)$ satisfies the PDE $\mu(j) G(t, x, j)+\frac{\partial G}{\partial t}(t, x, j)-\mu(j) x \frac{\partial G}{\partial x}(t, x, j)+\frac{1}{2} \sigma^{2}(j) x^{2} \frac{\partial^{2} G}{\partial x^{2}}(t, x, j)+\sum_{i=1}^{m} q_{j, i} G(t, x, i)=0$, and we conclude to (4.3) by (4.1). Next we note that (4.2) follows from

$$
\frac{\partial G}{\partial x}(t, x, j)=P\left(\frac{\hat{Y}_{t, T}}{Y_{t}}<x \mid \beta_{t}=j\right) \leq 1, \quad(t, x, j) \in[0, T] \times[1, \infty) \times \mathcal{M},
$$

cf. the definition (2.6) of $G$. Next, by (2.6) and Lemma 4.3, for any $(t, x, j) \in$ $[0, T] \times[1, \infty) \times \mathcal{M}$, we find

$$
\begin{aligned}
& \mathbb{L} G(t, x, j)=x \sigma^{2}(j) P\left(\frac{\hat{Y}_{t, T}}{Y_{t}}<x \mid \beta_{t}=j\right)-\mu(j) E\left[\max \left(x, \hat{Y}_{t, T} / Y_{t}\right) \mid \beta_{t}=j\right] \\
& =E\left[x \sigma^{2}(j) \mathbf{1}_{\left\{\hat{Y}_{t, T} / Y_{t}<x\right\}}-\mu(j)\left(x \vee \frac{\hat{Y}_{t, T}}{Y_{t}}\right) \mid \beta_{t}=j\right] \\
& =E\left[x\left(\sigma^{2}(j)-\mu(j)\right) \mathbf{1}_{\left\{\hat{Y}_{t, T} / Y_{t}<x\right\}} \mid \beta_{t}=j\right]-E\left[\mu(j)\left(x \vee \frac{\hat{Y}_{t, T}}{Y_{t}}\right) \mathbf{1}_{\left\{\hat{Y}_{t, T} / Y_{t} \geq x\right\}} \mid \beta_{t}=j\right],
\end{aligned}
$$

which shows (4.4), and implies by (4.8) that $\mathbb{L} G(t, x, j)$ is nondecreasing and continuous in $t \in[0, T]$ when $\mu(j) \geq 0$.

The proof of the next lemma, which will be used in Proposition 5.3 below, extends the argument of [2] page 993 to the regime-switching setting.

Lemma 4.4 We have

$$
\{(t, x, j) \in[0, T) \times[1, \infty) \times \mathcal{M}: \mathbb{L} G(t, x, j)<0\} \subset C
$$

where $C=D^{c}$ is the continuation set. 
Proof. By Lemma 4.7 below and Lemma 1 in [15] we have

$$
E\left[G\left(s, X_{s}^{t, x}, \beta_{s}\right) \mid \beta_{t}=j\right]=G(t, x, j)+E\left[\int_{t}^{s} \mathbb{L} G\left(r, X_{r}^{t, x}, \beta_{r}\right) d r \mid \beta_{t}=j\right],
$$

$s \in[t, T]$. Assume now that $(t, x, j) \in[0, T) \times[1, \infty) \times \mathcal{M}$ is such that $\mathbb{L} G(t, x, j)<0$. By the continuity of $\mathbb{L} G(t, x, j)$ with respect to $t$, there exists an open neighbourhood $U \subset[0, T) \times[1, \infty)$ of $(t, x)$ such that $\mathbb{L} G(s, y, j)<0$ for all $(s, y) \in U$. Replacing $s$ in (4.9) with the first exist time $\tau_{U}$ of $U$ when $\left(X_{s}^{t, x}, \beta_{s}\right)_{s \in[t, T]}$ is started at $(x, j)$ at time $t$, Relation (4.9) above shows by optional sampling that

$$
E\left[G\left(\tau_{U}, X_{\tau_{U}}^{t, x}, \beta_{\tau_{U}}\right) \mid \beta_{t}=j\right]=G(t, x, j)+E\left[\int_{t}^{\tau_{U}} \mathbb{L} G\left(r, X_{r}^{t, x}, \beta_{r}\right) d r \mid \beta_{t}=j\right] .
$$

Since $\tau_{U}>t$ a.s. and $\mathbb{L} G\left(r, X_{r}^{t, x}, \beta_{r}\right)<0$ when $r \in\left(t, \tau_{U}\right)$, the right hand side is strictly smaller than $G(t, x, j)$, while we have

$$
E\left[G\left(\tau_{U}, X_{\tau_{U}}^{t, x}, \beta_{\tau_{U}}\right) \mid \beta_{t}=j\right] \geq V(t, x, j)
$$

showing that $V(t, x, j)<G(t, x, j)$, which implies that $(t, x, j) \in C$.

Next we derive the following continuity result wich has been used in the proof of Proposition 3.1.

Lemma 4.5 For any $j \in \mathcal{M}$, the mapping $(t, x) \longmapsto V(t, x, j)$ is jointly continuous on $[0, T] \times[1, \infty)$.

Proof. We proceed in two steps. (i) We show that the mapping $t \longmapsto V(t, x, j)$ is continuous on $[0, T]$ for every fixed $x \geq 1$ and any $j \in \mathcal{M}$. By (2.4) we have

$$
\begin{aligned}
V(t, x, j) & =\inf _{t \leq \tau \leq T} E\left[\frac{\left(x Y_{t}\right) \vee \hat{Y}_{t, T}}{Y_{\tau}} \mid \beta_{t}=j\right] \\
& =\inf _{0 \leq \tau \leq T-t} E\left[\frac{x \vee \mathrm{e}^{0 \leq r \leq T-t} \max ^{\left(\left(\mu\left(\beta_{r}\right)-\sigma^{2}\left(\beta_{r}\right) / 2\right) r+\sigma\left(\beta_{r}\right) B_{r}\right)}}{\mathrm{e}^{\left(\mu\left(\beta_{\tau}\right)-\sigma^{2}\left(\beta_{\tau}\right) / 2\right) \tau+\sigma\left(\beta_{\tau}\right) B_{\tau}}} \mid \beta_{0}=j\right] \\
& =\inf _{0 \leq \tau \leq T-t} E\left[U(t, \tau) \mid \beta_{0}=j\right], \quad t \in[0, T], j \in \mathcal{M}, x \in[1, \infty),
\end{aligned}
$$

where

$$
U(t, s):=\frac{x \vee \mathrm{e}^{\max ^{0 \leq r \leq T-t}\left(\left(\mu\left(\beta_{r}\right)-\sigma^{2}\left(\beta_{r}\right) / 2\right) r+\sigma\left(\beta_{r}\right) B_{r}\right)}}{\mathrm{e}^{\left(\mu\left(\beta_{s}\right)-\sigma^{2}\left(\beta_{s}\right) / 2\right) \tau+\sigma\left(\beta_{s}\right) B_{s}}}, \quad s, t \in[0, T] .
$$




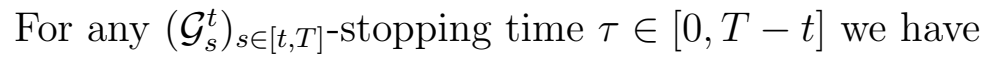

$$
\begin{aligned}
& 0 \leq E\left[U(t, \tau)-U(t+s, \tau) \mid \beta_{0}=j\right]
\end{aligned}
$$

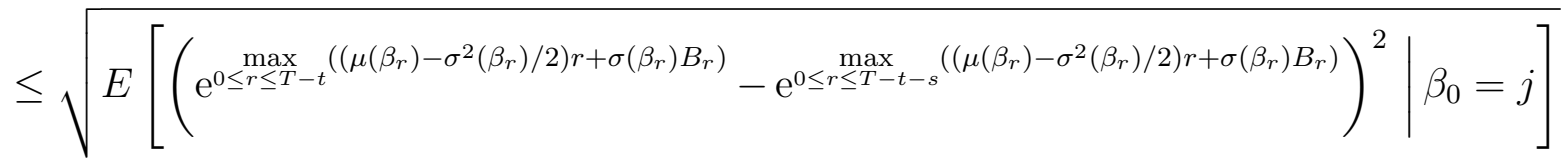

$$
\begin{aligned}
& \times \sqrt{E\left[\mathrm{e}^{-2\left(\mu\left(\beta_{\tau}\right)-\sigma^{2}\left(\beta_{\tau}\right) / 2\right) \tau-2 \sigma\left(\beta_{\tau}\right) B_{\tau}} \mid \beta_{0}=j\right]}
\end{aligned}
$$

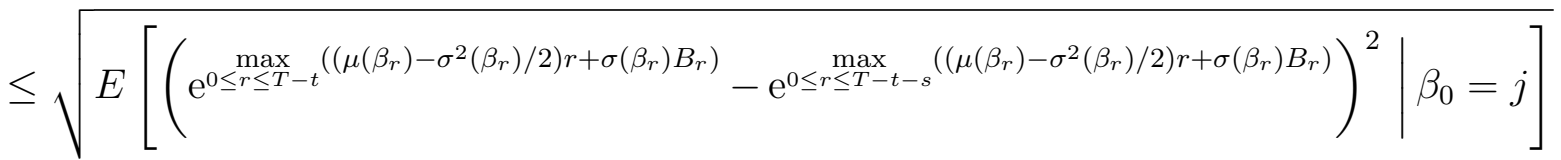

$$
\begin{aligned}
& \times \sqrt{\mathrm{e}^{(T-t) \max _{i \in \mathcal{M}}|3 \sigma(i)-2 \mu(i)|} E\left[\mathrm{e}^{-2 \sigma^{2}\left(\beta_{\tau}\right) \tau-2 \sigma\left(\beta_{\tau}\right) B_{\tau}} \mid \beta_{0}=j\right]}
\end{aligned}
$$

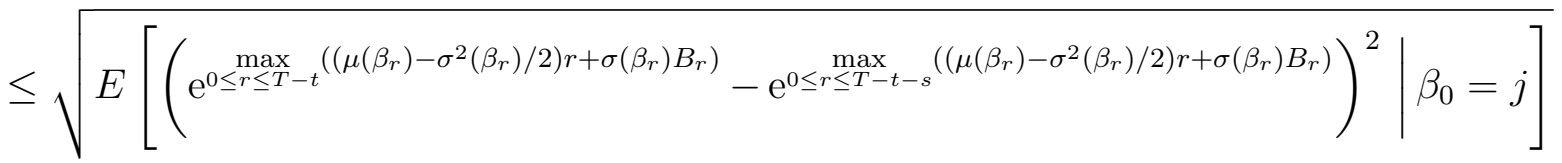

$$
\begin{aligned}
& \times \mathrm{e}^{(T-t) \max _{i \in \mathcal{M}}|3 \sigma(i)-2 \mu(i)| / 2},
\end{aligned}
$$

where we applied the optional sampling theorem. Letting $s$ tend to 0 on both sides of $(4.10)$, we get

$$
\lim _{s \searrow 0} E\left[U(t+s, \tau) \mid \beta_{0}=j\right]=E\left[U(t, \tau) \mid \beta_{0}=j\right]
$$

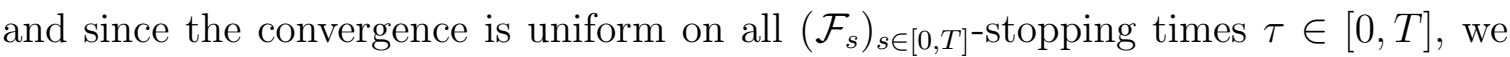
obtain

$$
\begin{aligned}
\liminf _{s \searrow 0} & \inf _{0 \leq \tau \leq T-t-s} E\left[U(t+s, \tau) \mid \beta_{0}=j\right] \geq \liminf _{s \searrow 0} \inf _{0 \leq \tau \leq T-t} E\left[U(t+s, \tau) \mid \beta_{0}=j\right] \\
= & \inf _{0 \leq \tau \leq T-t} \lim _{s \searrow 0} E\left[U(t+s, \tau) \mid \beta_{0}=j\right]=\inf _{0 \leq \tau \leq T-t} E\left[U(t, \tau) \mid \beta_{0}=j\right] . \quad \text { (4.11) }
\end{aligned}
$$

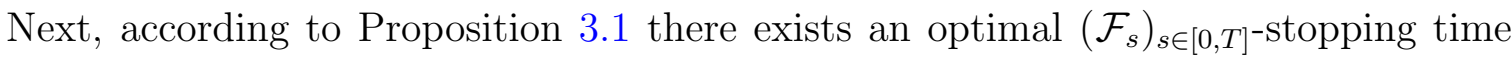
$\tau_{t}^{*} \in[0, T-t]$ such that

$$
\inf _{0 \leq \tau \leq T-t} E\left[U(t, \tau) \mid \beta_{0}=j\right]=E\left[U\left(t, \tau_{t}^{*}\right) \mid \beta_{0}=j\right]
$$

hence we have

$$
\inf _{0 \leq \tau \leq T-t-s} E\left[U(t+s, \tau) \mid \beta_{0}=j\right] \leq \inf _{0 \leq \tau \leq T-t-s} E\left[U(t, \tau) \mid \beta_{0}=j\right]
$$




$$
\leq E\left[U\left(t, \tau_{t}^{*} \wedge(T-t-s)\right) \mid \beta_{0}=j\right]
$$

Since $U(t, s)$ is nonnegative for any $s, t \in[0, T]$, we have

$$
\begin{aligned}
& U\left(t, \tau_{t}^{*} \wedge(T-t-s)\right) \leq U\left(t, \tau_{t}^{*}\right)+U(t, T-t-s) \\
& =U\left(t, \tau_{t}^{*}\right)+\frac{x \vee \mathrm{e}^{0 \leq r \leq T-t} \max ^{\left.\left.\left(\mu\left(\beta_{T-t-s}\right)-\sigma^{2}\left(\beta_{T-t-s}\right) / 2\right)(T-t-s)+\sigma\left(\beta_{T}\right)-\sigma^{2}\left(\beta_{r}\right) / 2\right) r+\sigma\left(\beta_{r}\right) B_{r}\right)}}{\mathrm{e}_{T-t-s}} \\
& \leq U\left(t, \tau_{t}^{*}\right)+\frac{x \vee \mathrm{e}^{0 \leq r \leq T-t} \max ^{\inf ^{i \in \mathcal{M}, r \in[0, T-t]}\left(\mu(i)-\sigma^{2}(i) / 2\right) r+} \inf _{i \in \mathcal{M}, r \in[0, T-t]}\left(\sigma(i) B_{r}\right)}{},
\end{aligned}
$$

which is integrable by (4.12). By the reverse Fatou Lemma we have

$$
\begin{gathered}
\limsup _{s \searrow 0} E\left[U\left(t, \tau_{t}^{*} \wedge(T-t-s)\right) \mid \beta_{0}=j\right] \leq E\left[\limsup _{s \searrow 0} U\left(t, \tau_{t}^{*} \wedge(T-t-s)\right) \mid \beta_{0}=j\right] \\
=E\left[U\left(t, \tau_{t}^{*}\right) \mid \beta_{0}=j\right] .
\end{gathered}
$$

Combining (4.12), (4.13), (4.14) and (4.11) we find

$$
\lim _{s \searrow 0} \inf _{0 \leq \tau \leq T-t-s} E\left[U(t+s, \tau) \mid \beta_{0}=j\right]=\inf _{0 \leq \tau \leq T-t} E\left[U(t, \tau) \mid \beta_{0}=j\right] .
$$

Similarly we have

$$
\lim _{s \searrow 0} \inf _{0 \leq \tau \leq T-t+s} E\left[U(t-s, \tau) \mid \beta_{0}=j\right]=\inf _{0 \leq \tau \leq T-t} E\left[U(t, \tau) \mid \beta_{0}=j\right],
$$

hence $t \longmapsto V(t, x, j)$ is continuous on $[0, T]$.

(ii) We show that $x \longmapsto V(t, x, j)$ is continuous on $[1, \infty)$, uniformly in $t \in[0, T]$, extending the argument of [2] page 995 to the regime-switching setting. By Relation (4.7) and the mean value theorem, for all $y \in[x, \infty)$ there exists a (random) $\eta \in\left[X_{t+\tau}^{t, x}, X_{t+\tau}^{t, y}\right]$ such that for any $\left(\mathcal{F}_{s}\right)_{s \in[0, T]}$-stopping time $\tau \in[0, T-t]$ we have

$$
\begin{gathered}
G\left(t+\tau, X_{t+\tau}^{t, y}, \beta_{t+\tau}\right)-G\left(t+\tau, X_{t+\tau}^{t, x}, \beta_{t+\tau}\right)=\frac{\partial G}{\partial x}(t+\tau, \eta)\left(X_{t+\tau}^{t, y}-X_{t+\tau}^{t, x}, \beta_{t+\tau}\right) \\
\leq(y-x) \frac{Y_{t}}{Y_{t+\tau}}
\end{gathered}
$$

since $X_{t+\tau}^{t, y}-X_{t+\tau}^{t, x} \leq(y-x) Y_{t} / Y_{t+\tau}$ by $(2.8)$. Let now $(t, x, j) \in[0, T] \times[1, \infty) \times \mathcal{M}$ and consider $\tau_{x}:=\tau(t, x, j)$ given by (3.2). By Lemma 2.2 we have

$$
V(t, y, j)-V(t, x, j) \leq E\left[G\left(t+\tau_{x}, X_{t+\tau_{x}}^{t, y}, \beta_{t+\tau_{x}}\right)-G\left(t+\tau_{x}, X_{t+\tau_{x}}^{t, x}, \beta_{t+\tau_{x}}\right) \mid \beta_{t}=j\right] .
$$


Since $E\left[Y_{t} / Y_{t+\tau} \mid \beta_{t}=j\right]$ is uniformly bounded as in (3.6), taking expectation on both sides of (4.15) yields

$$
\lim _{y \rightarrow x} E\left[G\left(t+\tau, X_{t+\tau}^{t, y}, \beta_{t+\tau}\right)-G\left(t+\tau, X_{t+\tau}^{t, x}, \beta_{t+\tau}\right) \mid \beta_{t}=j\right]=0
$$

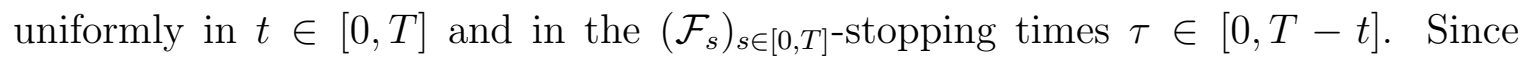
$V(t, x, j)$ is increasing in $x \in[1, \infty),(4.16)$ and (4.17) yield

$$
0 \leq \lim _{y \rightarrow x}(V(t, y, j)-V(t, x, j)) \leq 0
$$

which shows the continuity of $x \longmapsto V(t, x, j)$, uniformly in $t \in[0, T]$, for all $j \in \mathcal{M}$.

From $(i)$ and $(i i)$ we conclude to the joint continuity of $(t, x) \longmapsto V(t, x, j)$ on $[0, T] \times$ $[1, \infty)$ by classical arguments.

Lemma 4.6 The mapping $(t, x) \longmapsto G(t, x, j)$ is jointly continuous on $[0, T] \times[1, \infty)$.

Proof. By Relation (4.7) and the mean value theorem, for all $y \in[x, \infty)$ there exists an $\eta \in[x, y]$ such that for any $t \in[0, T]$ we have

$$
0 \leq G(t, y, j)-G(t, x, j)=(y-x) \frac{\partial G}{\partial x}(t, \eta, j) \leq y-x
$$

which shows the continuity of $x \longmapsto G(t, x, j)$, uniformly in $t \in[0, T]$. On the other hand, we have by $(2.6)$ that $t \longmapsto G(t, x, j)$ is continuous on $[0, T]$ for every $x \geq 1$. We conclude to the joint continuity of $(t, x) \longmapsto G(t, x, j)$ on $[0, T] \times[1, \infty)$ by a classical argument.

We close this section with the following lemma.

Lemma 4.7 The Markov process $\left(s, X_{s}^{t, x}, \beta_{s}\right)_{s \in[t, T]}$ has the infinitesimal generator

$$
\begin{aligned}
& \mathbb{L} f(s, y, j)=\left(\frac{\partial}{\partial s}+y\left(\sigma^{2}(j)-\mu(j)\right) \frac{\partial}{\partial y}+\frac{1}{2} \sigma^{2}(j) y^{2} \frac{\partial^{2}}{\partial y^{2}}\right) f(s, y, j)+\sum_{i=1}^{m} q_{j, i} f(s, y, i), \\
& s \in[0, T], j \in \mathcal{M}, y \in[1, \infty), \text { for } f \in \operatorname{Dom}(\mathbb{L}) \text { satisfying } \frac{\partial f}{\partial y}(s, 1+, j)=0 .
\end{aligned}
$$


Proof. Letting

$$
Z_{s}^{t, x}:=\log x \vee \hat{S}_{s-t}^{t}-\int_{0}^{s-t} \sigma\left(\beta_{u+t}\right) d \hat{B}_{u}^{t},
$$

$s \in[t, T], x \geq 1$, from (3.3) we have $X_{s}^{t, x}=\exp \left(Z_{s}^{t, x}\right), s \in[t, T], x \geq 1$. Since $\left(\hat{S}_{r}^{t}\right)_{r \in[0, T-t]}$ is nondecreasing it has finite variation, hence

$$
d\left\langle Z_{r}^{t, x}, Z_{r}^{t, x}\right\rangle=\sigma^{2}\left(\beta_{r}\right)\left\langle d \hat{B}_{r-t}^{t}, d \hat{B}_{r-t}^{t}\right\rangle=\sigma^{2}\left(\beta_{r}\right) d\left\langle B_{r}, B_{r}\right\rangle=\sigma^{2}\left(\beta_{r}\right) d r,
$$

which shows that

$$
\begin{aligned}
d X_{s}^{t, x} & =X_{s}^{t, x} d Z_{s}^{t, x}+\frac{1}{2} X_{s}^{t, x} d\left\langle Z_{s}^{t, x}, Z_{s}^{t, x}\right\rangle \\
& =X_{s}^{t, x} d Z_{s}^{t, x}+\frac{1}{2} \sigma^{2}\left(\beta_{s}\right) X_{s}^{t, x} d s \\
& =X_{s}^{t, x} d\left(\log x \vee \hat{S}_{s-t}^{t}\right)-\sigma\left(\beta_{s}\right) X_{s}^{t, x} d \hat{B}_{s-t}^{t}+\frac{1}{2} \sigma^{2}\left(\beta_{s}\right) X_{s}^{t, x} d s .
\end{aligned}
$$

Given that $\frac{\partial f}{\partial y}(s, 1+, j)=0$ for $(s, y, j) \in[0, T] \times[1, \infty) \times \mathcal{M}$, we have

$$
\begin{aligned}
\frac{\partial f}{\partial y}\left(s, X_{s}^{t, x}, \beta_{s}\right) d\left(\log x \vee \hat{S}_{s-t}^{t}\right) & =\frac{\partial f}{\partial y}\left(s, X_{s}^{t, x}, \beta_{s}\right) \mathbf{1}_{\left\{X_{s}^{t, x}>1\right\}} d\left(\log x \vee \hat{S}_{s-t}^{t}\right) \\
& =\frac{\partial f}{\partial y}\left(s, X_{s}^{t, x}, \beta_{s}\right) \mathbf{1}_{\left\{Z_{s}^{t, x}>0\right\}} d\left(\log x \vee \hat{S}_{s-t}^{t}\right) \\
& =0,
\end{aligned}
$$

since $d\left(\log x \vee \hat{S}_{s-t}^{t}\right)=0$ when $Z_{s}^{t, x}>0, s \in[t, T]$. From (4.18) this shows that

$$
\frac{\partial f}{\partial y}\left(s, X_{s}^{t, x}, \beta_{s}\right) d X_{s}^{t, x}=\frac{\partial f}{\partial y}\left(s, X_{s}^{t, x}, \beta_{s}\right)\left(-\sigma\left(\beta_{s}\right) X_{s}^{t, x} d \hat{B}_{s-t}^{t}+\frac{1}{2} \sigma^{2}\left(\beta_{s}\right) X_{s}^{t, x} d r\right),
$$

and we conclude the proof by Itô's calculus.

\section{Solution of the free boundary problem}

In this section we turn to the solution of the free boundary problem (2.4). We start by providing sufficient conditions on the drift coefficients $(\mu(j))_{j \in \mathcal{M}}$ for the boundary function $b_{D}(t, j)$ defined by (3.8) to be nonincreasing and continuous in $t \in[0, T]$. The next proposition 5.1 relies on Lemma 5.2 below. 
Proposition 5.1 Assume that $\mu(j) \geq 0$ for all $j \in \mathcal{M}$. Then the boundary function $b_{D}(t, j)$ defined by $(3.8)$ is nonincreasing in $t \in[0, T]$ and continuous in $t \in[0, T]$, for all $j \in \mathcal{M}$.

Proof. (i) Monotonicity. Let $(t, x, j) \in D$ and $s \in[t, T]$. We have $F(t, x, j)=0$ and $F(s, x, j)=0$ since $F(t, x, j)$ is nondecreasing in $t$ by Lemma 5.2 , hence

$$
[t, T] \times\{x\} \times\{j\} \subset D
$$

showing that $(t, x, j) \in D \Longleftrightarrow[t, T] \times\{x\} \times\{j\} \subset D$. Then for any $s \in(t, T]$, we have $\left(s, b_{D}(t, j), j\right) \in D$ since $\left(t, b_{D}(t, j), j\right) \in D$. By Proposition 3.2 and noting that $\left(s, b_{D}(s, j), j\right) \in D$, we conclude that $b_{D}(s, j) \leq b_{D}(t, j)$.

(ii) Right continuity. Given $\left(t, b_{D}(t, j), j\right) \in D$, consider a strictly decreasing sequence $\left(t_{n}\right)_{n \geq 1}$ such that $\lim _{n \rightarrow \infty} t_{n}=t$. By part $(i)$ above we know that $b_{D}\left(t_{n}, j\right) \leq b_{D}(t, j)$, $n \geq 1$, and $\lim _{n \rightarrow \infty} b_{D}\left(t_{n}, j\right) \leq b_{D}(t, j)$. Next, by Proposition 3.2 we have

$$
[t, T] \times\left[b_{D}(t, j), \infty\right) \times\{j\} \subset D
$$

and since $\left(t_{n}, j, b_{D}\left(t_{n}, j\right)\right) \in D, n \geq 1$, and $D$ is closed, we have $\left(t, \lim _{n \rightarrow \infty} b_{D}\left(t_{n}, j\right), j\right) \in$ $D$, hence $\lim _{n \rightarrow \infty} b_{D}\left(t_{n}, j\right) \geq b_{D}(t, j)$.

(iii) Left continuity. Using Lemma 4.4 we can repeat the argument of [2] page 998 provided we show that the function $h(t, j)$ defined by

$$
h(t, j):=\inf \{x \in[1, \infty): \mathbb{L} G(t, y, j) \geq 0, \quad \forall y \in[x, \infty)\}
$$

is continuous in $t \in[0, T]$ for all $j \in \mathcal{M}$, with $h(T, j)=1$. By Lemma 4.3 the function $\mathbb{L} G(t, x, j)$ is nondecreasing in $t$ for all $x \geq 1$ since $(j) \geq 0$ and it follows from the definition (5.1) of $h(t, j)$ that $t \longmapsto h(t, j)$ is nonincreasing in $t \in[0, T]$. For any $t_{0} \in[0, T)$ and decreasing sequence $\left(t_{n}\right)_{n \geq 1} \subset\left(t_{0}, T\right]$ converging $t_{0}$ from the righthand side we have $\lim _{n \rightarrow \infty} h\left(t_{n}, j\right) \leq h\left(t_{0}, j\right)$ and $\lim _{n \rightarrow \infty} h\left(t_{n}, j\right) \geq h\left(t_{k}, j\right)$ for any $k \geq 1$, hence $\lim _{n \rightarrow \infty} h\left(t_{n}, j\right) \geq h\left(t_{0}, j\right)$ as by the continuity of $t \rightarrow \mathbb{L} G(t, x, j)$ we have

$$
\mathbb{L} G\left(t_{0}, \lim _{n \rightarrow \infty} h\left(t_{n}, j\right), j\right)=\lim _{k \rightarrow \infty} \mathbb{L} G\left(t_{k}, \lim _{n \rightarrow \infty} h\left(t_{n}, j\right), j\right) \geq 0,
$$

and this proves that $\lim _{t \searrow t_{0}} h(t, j)=h\left(t_{0}, j\right)$. On the other hand we have $h\left(t_{0}-, j\right):=$ $\lim _{t \uparrow t_{0}} h(t, j) \geq h\left(t_{0}, j\right)$ for any $t_{0} \in[0, T], j \in \mathcal{M}$. In case $h\left(t_{0}-, j\right)>h\left(t_{0}, j\right)$ we 
have $\mathbb{L} G\left(t_{0}, x, j\right) \geq 0$ for all $x \in\left[h\left(t_{0}, j\right), \infty\right]$. In addition, for any $t \in\left[0, t_{0}\right)$ and $x \in\left[h\left(t_{0}, j\right), h\left(t_{0}-, j\right)\right)$ we have $\mathbb{L} G(t, x, j)<0$ since $h(t, j) \geq h\left(t_{0}-, j\right)$, hence $\mathbb{L} G\left(t_{0}, x, j\right)=0$ for all $x \in\left[h\left(t_{0}, j\right), h\left(t_{0}-, j\right)\right)$ by the continuity of $t \longmapsto \mathbb{L} G(t, x, j)$. By Lemma 4.3 we would have

$$
x \sigma^{2}(j) \frac{\partial G}{\partial x}\left(t_{0}, x, j\right)=\mu(j) G\left(t_{0}, x, j\right), \quad x \in\left[h\left(t_{0}, j\right), h\left(t_{0}-, j\right)\right),
$$

which shows that $G\left(t_{0}, x, j\right)=C\left(t_{0}, j\right) x^{\mu(j) / \sigma^{2}(j)}$, where $C\left(t_{0}, j\right)$ depends only on $t_{0}$ and $j \in \mathcal{M}$. This is a contradiction since $\frac{\partial}{\partial x} G\left(t_{0}, x, j\right)=P\left(\hat{Y}_{t_{0}, T} / Y_{t_{0}}<x \mid \beta_{t_{0}}=j\right)=$ $C\left(t_{0}, j\right) \mu(j) x^{-1+\mu(j) / \sigma^{2}(j)} / \sigma^{2}(j)$ for $x \in\left[h\left(t_{0}, j\right), h\left(t_{0}-, j\right)\right)$ cannot hold when $\mu(j)<$ $\sigma^{2}(j)$, and more generally $\hat{Y}_{t_{0}, T} / Y_{t_{0}}$ cannot have a power law, even locally.

Figure 1 illustrates the result of Proposition 5.1 by applying the recursive algorithm of [9] in order to plot the value functions $V(t, a, j)$ and $G(t, a, j)$. In Figure 1 we take the positive drifts $\mu(1)=0.15, \mu(2)=0.05$, with $\sigma(1)=0.5, \sigma(2)=0.3, T=0.5$, $n=100, \delta_{n}=T / n=0.05$, and

$$
\mathbf{Q}=\left[\begin{array}{cc}
-2.5 & 2.5 \\
2 & -2
\end{array}\right]
$$
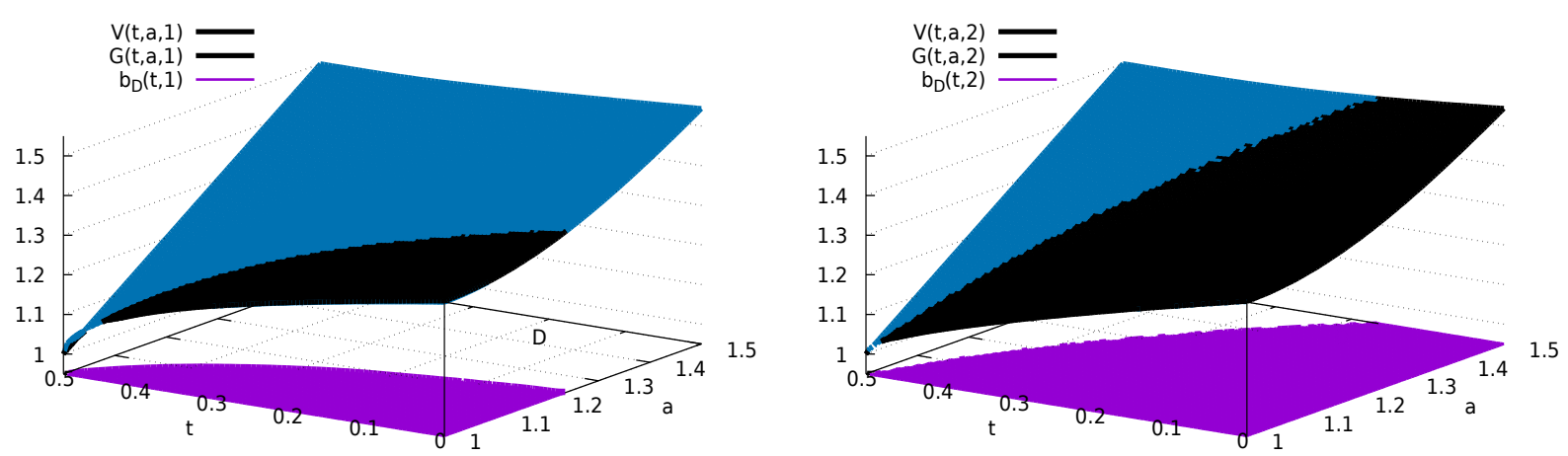

Figure 1: Value functions in the two-state case.

Figure 1 also allows us to visualize the stopping set $D$ and the continuation set

$$
C=\{(t, a, j) \in[0, T] \times[1, \infty) \times \mathcal{M}: V(t, a, j)<G(t, a, j)\} .
$$

The numerical instabilities observed are due to the necessity to check the equality $V(t, a, j)=G(t, a, j)$ when $V(t, a, j)$ and $G(t, a, j)$ are very close to each other. 


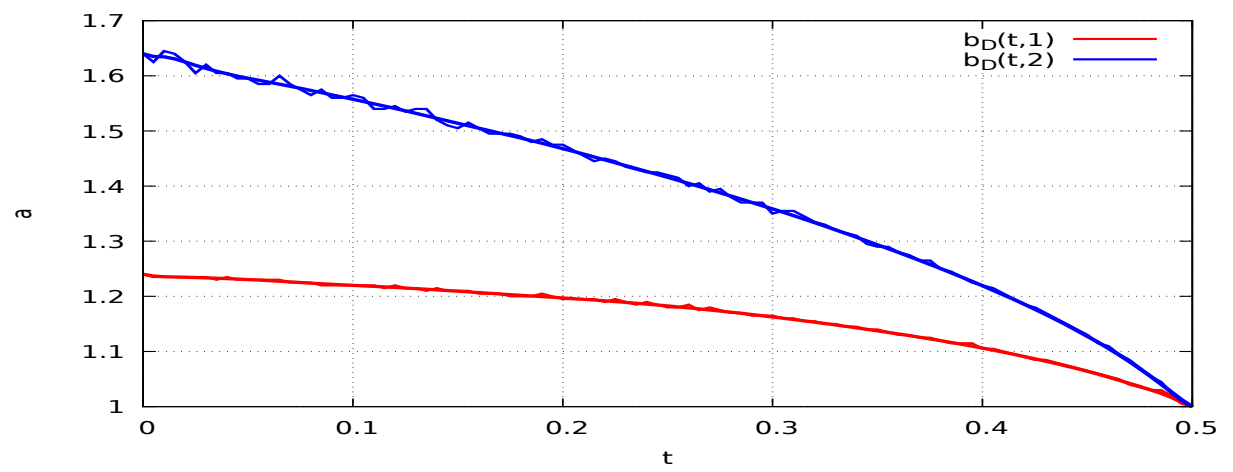

Figure 2: Boundary functions in the two-state case.

The boundary functions are plotted in Figure 2 based on Figure 1, with spline smoothing. We observe that starting from state 1 it is better to exercise earlier than if we start from state 2 which has a lower drift. This is due to the possibility to switch from state 1 to state 2 after the average time $1 / q_{1,1}=0.4$ and to stay at state 2 for the remaining time $T-t \leq 1 / q_{2,2}=0.5$, in which case the drift takes the lower value $\mu(2)=0.05$. The opposite occurs if we start from state 2 , for which the boundary graph is higher than if we start from state 1.

Similarly to (3.22)-(3.23) in [2], we now show that $F(t, x, j)$ defined by (3.7) is nondecreasing in $t \in[0, T]$ for all $j \in \mathcal{M}$ and $x \in[1, \infty)$, as in the following Lemma 5.2 which has been used for Proposition 5.1, and whose proof follows [2] page 994.

We note that without the condition $\mu(i) \geq 0$ for all $i \in \mathcal{M}$, the function $F(t, x, j)$ in Lemma 5.2 may not be nondecreasing in $t \in[0, T]$, in which case the equivalence $(t, x, j) \in D \Longleftrightarrow[t, T] \times\{x\} \times\{j\} \subset D$ in the proof of the next Proposition 5.1 does not hold and in this situation the boundary function $t \longmapsto b_{D}(t, j)$ may not be decreasing in $t \in[0, T]$, cf. Figure 4 in [9].

Lemma 5.2 Under the condition $\mu(j) \geq 0$ for all $j \in \mathcal{M}$, the function

$$
t \longmapsto F(t, x, j)=V(t, x, j)-G(t, x, j)
$$

is nondecreasing in $t \in[0, T]$, for any $(j, x) \in \mathcal{M} \times[1, \infty)$. 
Proof. For any $r, s \in[0, T-t]$, and $r<s$, denote $\tau_{s}:=\tau_{D}(s, x, j)-s \in[0, T-s]$ by the definition (3.2) of $\tau_{D}$. Replacing $s$ with $\tau_{s}$ and $t$ with $r$ in the formula (4.9), and using optional sampling, we have

$$
\begin{aligned}
F(r, x, j) & =V(r, x, j)-G(r, x, j) \\
& \leq E\left[G\left(r+\tau_{s}, X_{r+\tau_{s}}^{r, x}, \beta_{r+\tau_{s}}\right) \mid \beta_{r}=j\right]-G(r, x, j) \\
& =E\left[\int_{r}^{r+\tau_{s}} \mathbb{L} G\left(v, X_{v}^{r, x}, \beta_{v}\right) d v \mid \beta_{r}=j\right] \\
& =E\left[\int_{0}^{\tau_{s}} \mathbb{L} G\left(v+r, X_{v+r}^{r, x}, \beta_{v+r}\right) d v \mid \beta_{r}=j\right] \\
& =E\left[\int_{0}^{\tau_{s}} \mathbb{L} G\left(v+r, X_{v}^{0, x}, \beta_{v}\right) d v \mid \beta_{0}=j\right] .
\end{aligned}
$$

Combining (5.2) with

$$
\begin{gathered}
F(s, x, j)=V(s, x, j)-G(s, x, j)=E\left[G\left(s+\tau_{s}, X_{s+\tau_{s}}^{r, x}, \beta_{s+\tau_{s}}\right) \mid \beta_{r}=j\right]-G(s, x, j) \\
=E\left[\int_{0}^{\tau_{s}} \mathbb{L} G\left(v+s, X_{v}^{0, x}, \beta_{v}\right) d v \mid \beta_{0}=j\right]
\end{gathered}
$$

we have

$$
\begin{aligned}
& F(s, x, j)-F(r, x, j) \\
& \quad \geq E\left[\int_{0}^{\tau_{s}} \mathbb{L} G\left(v+s, X_{v}^{0, x}, \beta_{v}\right) d v \mid \beta_{0}=j\right]-E\left[\int_{0}^{\tau_{s}} \mathbb{L} G\left(v+r, X_{v}^{0, x}, \beta_{v}\right) d v \mid \beta_{0}=j\right] \\
& \quad=E\left[\int_{0}^{\tau_{s}} \mathbb{L} G\left(v+s, X_{v}^{0, x}, \beta_{v}\right)-\mathbb{L} G\left(v+r, X_{v}^{0, x}, \beta_{v}\right) d v \mid \beta_{0}=j\right] .
\end{aligned}
$$

Since by (4.8) the function $t \mapsto \mathbb{L} G(t, x, i)$ is nondecreasing in $t$ when $\mu(i) \geq 0$, we find that the right hand side of $(5.3)$ is nonnegative, thereby $F(t, x, j)$ is nondecreasing in $t \in[0, T]$.

\section{Particular exercise strategies}

Next we show that the stopping set $D$ has a simple form in two special situations.

Proposition 5.3 We have the following special cases of optimal stopping sets D.

i) Immediate exercise. Under the condition $\mu(j) \leq 0$ for all $j \in \mathcal{M}$, we have $D=[0, T] \times[1, \infty) \times \mathcal{M}$. 
ii) Exercise at maturity. Under the condition $\mu(j) \geq \sigma^{2}(j)$ for all $j \in \mathcal{M}$, we have $D=\{T\} \times[1, \infty) \times \mathcal{M}$.

Proof. Replacing $s$ in (4.9) with $\tau_{D}$ defined in (3.2) and using optional sampling, we find

$$
V(t, x, i)=G(t, x, i)+E\left[\int_{t}^{\tau_{D(t, x, i)}} \mathbb{L} G\left(r, X_{r}^{t, x}, \beta_{r}\right) d r \mid \beta_{t}=i\right], \quad t \in[0, T] .
$$

i) In case $\mu(j) \leq 0$ for all $j \in \mathcal{M}$, by Lemma 4.3 , we have $\mathbb{L} G(t, x, i)>0$ for all $(t, x, i) \in[0, T) \times[1, \infty) \times \mathcal{M}$, hence (5.4) implies $\tau_{D}(t, x, i)=0$ a.s., otherwise it contradicts the fact that $V(t, x, i) \leq G(t, x, i)$ because of (5.4). This implies $[0, T] \times[1, \infty) \times \mathcal{M} \subset D$.

ii) In case $\mu(j) \geq \sigma^{2}(j)$ for all $j \in \mathcal{M}$, by Lemma 4.3 we have $\mathbb{L} G(t, x, i)<0$ for all $(t, x, i) \in[0, T) \times[1, \infty) \times \mathcal{M}$, and applying Lemma 4.4, we see that $[0, T) \times[1, \infty) \times \mathcal{M} \subset C$, which means $D=\{T\} \times[1, \infty) \times \mathcal{M}$.

Finally we derive a Volterra type equation (5.5) below satisfied by the function $b_{D}\left(t, \beta_{t}\right)$ defined in (3.8), for the boundary curves

$$
\left\{(t, x) \in[0, T] \times[1, \infty): x=b_{D}(t, j)\right\}
$$

of the optimal stopping set $D$ in (3.1), for any $j \in \mathcal{M}$.

Proposition 5.4 Assume that $\mu(j) \geq 0, j \in \mathcal{M}$. The boundary function $b_{D}(t, j)$ satisfies the Volterra type equation

$$
G\left(t, b_{D}(t, j), j\right)=J\left(t, b_{D}(t, j), j\right)-\int_{t}^{T} K\left(t, r, b_{D}(t, j), j\right) d r
$$

$0 \leq t \leq T$, with terminal condition $b_{D}(T, j)=1, j \in \mathcal{M}$, where

$$
J(t, x, j):=E\left[X_{T}^{t, x} \mid \beta_{t}=j\right],
$$

and

$$
K(t, r, x, j):=E\left[\mathbb{L} V\left(r, X_{r}^{t, x}, \beta_{r}\right) \mathbf{1}_{\left\{X_{r}^{t, x}>b_{D}\left(r, \beta_{r}\right)\right\}} \mid \beta_{t}=j\right],
$$

for $0 \leq t \leq r \leq T$ and $x \geq 1$. 
Proof. Noting that $V(t, x, j) \leq G(t, x, j)$ for all $(t, x, j) \in[0, T] \times[1, \infty) \times \mathcal{M}$ by (2.7), the continuation set $C:=D^{c}$ is given by

$$
C=D^{c}=\{(t, x, j) \in[0, T] \times[1, \infty) \times \mathcal{M}: V(t, x, j)<G(t, x, j)\} .
$$

According to Proposition 3.1, for any $(t, x, j) \in C$, we have

$$
V(t, x, j)=E\left[G\left(\tau_{D}, X_{\tau_{D}}^{t, x}, \beta_{\tau_{D}}\right) \mid \beta_{t}=j\right]
$$

where $\tau_{D}=\tau_{D}(t, x, j)$ is defined by (3.2). Given that $\frac{\partial V}{\partial y}(t, 1+, j)=0$ by Lemma 4.2 , by the application of [11], Chapter III, $\S 7.1 .1, \S 7.4 .1$ as in [2] $\S 3.5$, page 996, the function $V$ in (5.9) is $\mathcal{C}^{1,2}$ in the continuation set $C$ in (5.8) and it solves the Cauchy-Dirichlet free boundary problem

$$
\begin{cases}\mathbb{L} V(t, y, j)=0, & (t, y, j) \in C, \\ V(t, y, j)=G(t, y, j), & (t, y, j) \in \partial C,\end{cases}
$$

hence $\partial C \subset D$, where $\partial C$ denotes the boundary of the open set $C$. By the local time change of variable formula of [10], and by Lemma 4.7 below with the property $\frac{\partial V}{\partial y}(t, 1+, j)=0$ shown in Lemma 4.2 above, we have

$$
\begin{aligned}
& E\left[X_{T}^{t, x} \mid \beta_{t}=j\right]=E\left[V\left(T, X_{T}^{t, x}, \beta_{T}\right) \mid \beta_{t}=j\right] \\
& \quad=V(t, x, j)+E\left[\int_{t}^{T} \mathbb{L} V\left(r, X_{r}^{t, x}, \beta_{r}\right) \mathbf{1}_{\left\{X_{r}^{t, x} \neq b_{D}\left(r, \beta_{r}\right)\right\}} d r \mid \beta_{t}=j\right] \\
& +\frac{1}{2} E\left[\int_{t}^{T}\left(\frac{\partial V}{\partial y}\left(r, X_{r}^{t, x}+, \beta_{r}\right)-\frac{\partial V}{\partial y}\left(r, X_{r}^{t, x}-, \beta_{r}\right)\right) \mathbf{1}_{\left\{X_{r}^{t, x}=b_{D}\left(r, \beta_{r}\right)\right\}} d \ell_{r}^{b}\left(X^{t, x}\right) \mid \beta_{t}=j\right],
\end{aligned}
$$

where we applied the equality $V\left(T, X_{T}^{t, x}, \beta_{T}\right)=X_{T}^{t, x}$, and $\left(\ell_{r}^{b}\left(X^{t, x}\right)\right)_{r \in[t, T]}$ denotes the local time of $X^{t, x}$ on the (piecewise continuous and nonincreasing by Proposition 5.1) curve $r \longmapsto b_{D}\left(r, \beta_{r}\right)$. By the smooth fit property shown in Lemma 4.1 above, the last term in (5.11) vanishes. By Proposition 3.2 above and the definition $(3.8)$ of $b_{D}(t, j)$, Relation (5.10a) can be rewritten as

$$
\mathbb{L} V(r, y, j) \mathbf{1}_{\left\{y<b_{D}(r, j)\right\}}=0, \quad r \in[0, T], j \in \mathcal{M}, y \geq 1,
$$


which implies

$E\left[\int_{t}^{T} \mathbb{L} V\left(r, X_{r}^{t, x}, \beta_{r}\right) d r \mid \beta_{t}=j\right]=E\left[\int_{t}^{T} \mathbb{L} V\left(r, X_{r}^{t, x}, \beta_{r}\right) \mathbf{1}_{\left\{X_{r}^{t, x}>b_{D}\left(r, \beta_{r}\right)\right\}} d r \mid \beta_{t}=j\right]$.

Hence, combining (5.11) and (5.12), we obtain

$$
E\left[X_{T}^{t, x} \mid \beta_{t}=j\right]=V(t, x, j)+\int_{t}^{T} E\left[\mathbb{L} V\left(r, X_{r}^{t, x}, \beta_{r}\right) \mathbf{1}_{\left\{X_{r}^{t, x}>b_{D}\left(r, \beta_{r}\right)\right\}} \mid \beta_{t}=j\right] d r
$$

and substituting $x$ with $b_{D}(t, j)$ in (5.13) above we find that

$$
\begin{aligned}
& G\left(t, b_{D}(t, j), j\right)=V\left(t, b_{D}(t, j), j\right) \\
& \quad=E\left[X_{T}^{t, b_{D}(t, j)} \mid \beta_{t}=j\right]-E\left[\int_{t}^{T} \mathbb{L} V\left(r, X_{r}^{t, b_{D}(t, j)}, \beta_{r}\right) \mathbf{1}_{\left\{X_{r}^{t, x} \geq b_{D}\left(r, \beta_{r}\right)\right\}} d r \mid \beta_{t}=j\right] \\
& \quad=J\left(t, b_{D}(t, j), j\right)-\int_{t}^{T} K\left(t, r, b_{D}(t, j), j\right) d r,
\end{aligned}
$$

where the functions $J, K$ are defined by (5.6)-(5.7).

Remark 5.5 Note that the equation (5.5) also involves the optimal value function $V(r, y, j)$ and not only the function $G(r, y, j)$. Indeed, when $m \geq 2$ the equality $V(r, y, j)=G(r, y, j)$ in (5.12) for a given $(r, y, j)=\left(r, X_{r}^{t, x}, \beta_{r}\right) \in D$ does not imply

$$
\mathbb{L} V(r, y, j)=\mathbb{L} G(r, y, j)
$$

as in [2] because we may not have $V(r, y, i)=G(r, y, i)$ for all $i=1, \ldots, m$ in the summation over the states of $\left(\beta_{t}\right)_{t \in[0, T]}$ in the definition (4.1) of $\mathbb{L}$. In [1] this issue is dealt with via an ordering assumption on the boundary functions $\left(b_{D}(t, j)\right)_{t \in[0, T]}$ in the two-state case $j=1,2$, see Assumption 3.1 therein, however this method applies specifically to American options and not to ultimate maximum problems, which have a more complex payoff structure. Moreover, such an ordering condition may not be satisfied in our current setting, cf. Figure 4 of [9].

In the absence of regime switching with $Y_{t}=Y_{0} \mathrm{e}^{\left(\mu-\sigma^{2} / 2\right) t+\sigma B_{t}}$, Relation (3.8) is replaced by

$$
b_{D}(t)=\inf \left\{x \in \mathbb{R}_{+}:(t, x) \in D\right\}, \quad t \in[0, T]
$$


and the boundary equation (5.5) becomes

$$
G\left(t, b_{D}(t)\right)=E\left[X_{T}^{t, b_{D}(t)}\right]-E\left[\int_{t}^{T} \mathbb{L} G\left(r, X_{r}^{b_{D}(t)}\right) \mathbf{1}_{\left\{X_{r}^{t, b_{D}(t)}>b_{D}(r)\right\}} d r\right],
$$

which recovers $(3.50)$ in [2], with

$$
\mathbb{L} G(r, x)=\left(\frac{\partial}{\partial r}+x\left(\sigma^{2}-\mu\right) \frac{\partial}{\partial x}+\frac{1}{2} \sigma^{2} x^{2} \frac{\partial^{2}}{\partial x^{2}}\right) G(r, x), \quad r \in[0, T], x \in \mathbb{R}_{+} .
$$

Since the Volterra type equation (5.5) cannot be solved by standard methods under regime switching, we have applied the recursive algorithm of [9] in order to plot Figures 1 and 2 .

\section{References}

[1] J. Buffington and R.J. Elliott. American options with regime switching. Int. J. Theor. Appl. Finance, 5(5):497-514, 2002.

[2] J. du Toit and G. Peskir. Selling a stock at the ultimate maximum. Ann. Appl. Probab., 19(3):983-1014, 2009.

[3] P. Eloe, R. H. Liu, M. Yatsuki, G. Yin, and Q. Zhang. Optimal selling rules in a regime-switching exponential Gaussian diffusion model. SIAM J. Appl. Math., 69(3):810-829, 2008.

[4] X. Guo. An explicit solution to an optimal stopping problem with regime switching. J. Appl. Probab., 38(2):464-481, 2001.

[5] X. Guo and Q. Zhang. Optimal selling rules in a regime switching model. IEEE Trans. Automat. Control, 50(9):1450-1455, 2005.

[6] J.D. Hamilton. A new approach to the economic analysis of non-stationary time series. Econometrica, 57:357-384, 1989.

[7] H. Le and C. Wang. A finite time horizon optimal stopping problem with regime switching. SIAM J. Control Optim., 48(8):5193-5213, 2010.

[8] R H. Liu, Q. Zhang, and G. Yin. Option pricing in a regime-switching model using the fast Fourier transform. J. Appl. Math. Stoch. Anal., pages Art. ID 18109, 22, 2006.

[9] Y. Liu and N. Privault. A recursive algorithm for selling at the ultimate maximum in regimeswitching models. Preprint, 18 pages, 2015.

[10] G. Peskir. A change-of-variable formula with local time on curves. J. Theoret. Probab., 18(3):499-535, 2005.

[11] G. Peskir and A. Shiryaev. Optimal stopping and free-boundary problems. Lectures in Mathematics ETH Zürich. Birkhäuser Verlag, Basel, 2006.

[12] A.N. Shiryaev. Optimal stopping rules. Springer-Verlag, New York, NY, 1978.

[13] A.N. Shiryaev, Z. Xu, and X.Y. Zhou. Thou shalt buy and hold. Quant. Finance, 8(8):765-776, 2008. 
[14] V. Ly Vath and H. Pham. Explicit solution to an optimal switching problem in the two-regime case. SIAM J. Control Optim., 46(2):395-426, 2007.

[15] D.D. Yao, Q. Zhang, and X.Y. Zhou. A regime-switching model for European options. International Series in Operation Research and Management Science, 94:281-300, 2006. 